A) Check for updates

Cite this: Org. Biomol. Chem., 2019, 17, 4102

Received 13th February 2019,

Accepted 2nd April 2019

DOI: 10.1039/c9ob00357f

rsc.li/obc

\section{Synthesis and characterization of propeller-shaped mono- to hexacationic quinolinium-substituted benzenes $\uparrow$}

\author{
Sviatoslav Batsyts, (D) a Eike G. Hübner, (D) a Jan C. Namyslo, ${ }^{a}$ Mimoza Gjikaj ${ }^{\mathrm{b}}$ and \\ Andreas Schmidt (iD *a
}

\begin{abstract}
Diels-Alder reaction of 2-, 3- and 4-(phenylethynyl)quinolines and tetraphenylcyclopentadienone gave three regioisomeric 2,3,4,5,6-pentaphenyl-1-(quinolin-2-yl, -3-yl, and -4-yl)benzenes. Restricted rotation of the 3-yl and 4-yl substituted derivatives is observed between the central core and the substituents, resulting in propeller-shaped molecules. Likewise, 1,2-diquinolinyl-3,4,5,6-tetraphenylbenzenes with 3-yl,3-yl and 3-yl,4-yl connectivity were prepared. As evidenced by NMR spectroscopy, they form two diasteromers due to their restricted rotation. A cobalt-catalyzed [2+2+2]-cyclotrimerization of 2-(phenylethynyl)quinoline resulted in the formation of triphenyl-2,4,6- and -3,5,6-tri(quinolin-2-yl)benzenes. The same reaction was applied to 3,3'-ethyne-1,2-diyldiquinoline which formed hexa(quinolin-3-yl) benzene. $\mathrm{N}$-Methylation gave the title compounds. Among those, the hexacationic hexa( $N$-methylquinolinio-3-yl)benzene is described. Stereochemical aspects are predominantly discussed by means of results of NMR experiments. DFT-calculations on the most stable conformations and the frontier orbital profiles of the hexacation as well as of its neutral precursor have been carried out.
\end{abstract}

\section{Introduction}

Considerable attention has been directed toward heterocycle polycations as they are interesting in natural product chemistry, heterocyclic chemistry, and materials chemistry. Thus, polypyridinium alkaloids (halitoxin, ${ }^{1}$ cyclostellettamin $\mathrm{C},{ }^{2}$ amphitoxin, ${ }^{3}$ viscosamine, ${ }^{4}$ viscosaline, ${ }^{5}$ pachychalines $\mathrm{A}-\mathrm{C}^{6}$ ) are polycationic molecules from nature. Streitwieser et al. reported on series of polypyridinium salts and betaines which have adjacent heteroarenium rings in conjugation. ${ }^{7}$ Thus, the five-fold pyridinium substituted cyclopentadiene anion $1^{8}$ possesses structure elements of conjugated mesomeric betaines. The SASAPOS protocol (self-activated silyl-assisted polyonio substitution) by Weiss et al. allowed for the synthesis of a variety of heteroarenium substituted substrates, for which 2 is given as an example here. ${ }^{9}$ This widely applicable protocol ${ }^{10}$ takes advantage of the fact that a substrate which bonds neutral ligands such as chloride undergoes a substitution with heteroaromatic nucleophiles equivalent to the quantity of the

${ }^{a}$ Clausthal University of Technology, Institute of Organic Chemistry, Leibnizstraße 6, D-38678 Clausthal-Zellerfeld, Germany.E-mail: schmidt@ioc.tu-clausthal.de

${ }^{b}$ Clausthal University of Technology, Institute of Inorganic and Analytical Chemistry, Paul-Ernst-Straße 4, D-38678 Clausthal-Zellerfeld, Germany

$\dagger$ Electronic supplementary information (ESI) available: Calculations, NMR spectra. See DOI: 10.1039/c9ob00357f bonding ligands in the presence of the same number of equivalents of trimethylsilyltriflate (TMSOTf). We reported on heterocycle polycations with heteroaromatic central cores such as pyridine ${ }^{11}$ pyrimidine, ${ }^{12}$ pyrazine, ${ }^{13}$ pyridazine, ${ }^{13} 1,3,5$-triazine, ${ }^{13}$ and purine. ${ }^{13}$ An example is pentacation $3 .{ }^{14}$ Pyridine polycations proved to be versatile starting materials for the synthesis of highly substituted pyridines with various substitution patterns. ${ }^{11}$ Apart from their synthetic applicabilities, heterocycle polycations are of interest as potential semiconductors, ${ }^{15}$ photosensitive materials, ${ }^{16}$ oxidants, ${ }^{17}$ and biologically active compounds like herbicides, ${ }^{18}$ acetylcholinesterase reactivators ${ }^{19}$ and cholinesterase inhibitors. ${ }^{20}$ A recent review article summarizes results achieved so far (Scheme 1). ${ }^{21}$

Steric hindrance exerted by the peripheral ligands cause propeller-shaped molecules. Recently, the sterically congested hexa(heteroaryl)benzene (HHAB) 4 was formed as two isolable isomers which differ only in the rotation of one methyl-pyridine group. ${ }^{22}$ In general, propeller-shaped hexaarylbenzenes (HABs) such as hexa( $\beta$-naphthyl)benzene $5^{23}$ have numerous applications in materials sciences as they play roles as liquid crystals, ${ }^{24}$ microporous organic solids, ${ }^{25}$ molecular capsules, ${ }^{26}$ supramolecular electronic materials, ${ }^{27}$ molecular rotors, ${ }^{28}$ nonlinear optical materials, ${ }^{29}$ metal sensors, ${ }^{30}$ redox materials, ${ }^{31}$ and molecular wires. ${ }^{32}$ We report here on quinolinium-substituted benzenes which combine the features of HHABs and polycations as they have to adopt propeller-shaped 


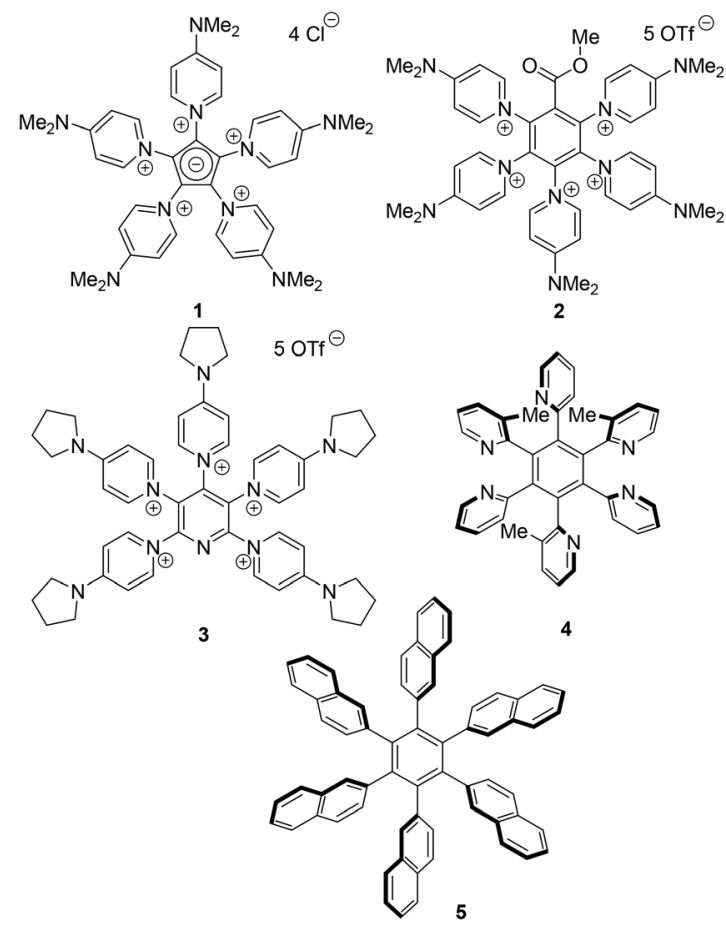

Scheme 1 Examples of heterocycle polycations and of propellershaped molecules.

configurations due to the additional benzo-annulation in comparison to their pyridinium derivatives.

\section{Results and discussion}

First, Sonogashira-Hagihara coupling reactions were used to prepare three isomeric ethynyl-substituted quinolines as starting materials for the synthesis of monoquinolinyl-substituted pentaphenylbenzenes, which are of potential interest as ligands of organic electroluminescent device materials. ${ }^{33}$ Thus, the 2-, 3-, and 4-halosubstituted quinolines $\mathbf{6 a - c}$ and phenylacetylene 7 were reacted to give the quinolines $\mathbf{8 a - c}$ in good yields (Scheme 2). With these compounds in hand, we

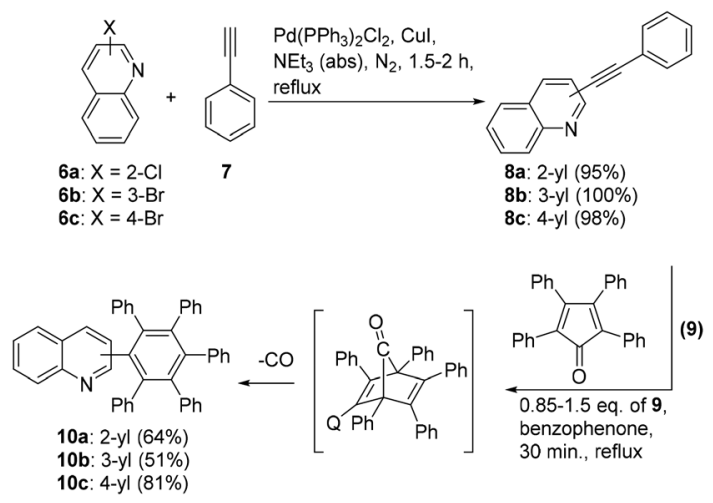

Scheme 2 Synthesis of monoquinoline-substituted HHABs. examined the $[4+2]$-cycloaddition with tetraphenylcyclopentadione 9. The reaction did not proceed under a variety of different conditions that were tested, among those reflux temperature in benzene, toluene, and xylene, respectively. On increasing the reaction temperature to $305{ }^{\circ} \mathrm{C}$ by using benzophenone as a solvent, however, the quinolines 10a-c were finally prepared within $30 \mathrm{~min}$ in moderate yields.

Similar to hexaphenylbenzene, the six peripheral rings of 10a-c cannot lie in the plane of the central benzene ring. Whereas in solution, on the NMR time scale, the peripheral rings are perpendicular to the plane of the central ring in the absence of appropriate substitutions, the X-ray structure of hexaphenylbenzene itself showed a propeller conformation with angles around the $\mathrm{Ph}-\mathrm{Ph}$ bonds of approximately $65^{\circ} .^{34} \mathrm{It}$ is known that methyl and methoxy groups in $\mathrm{C}_{6} \mathrm{Ar}_{6}$ systems in ortho position cause a barrier of rotation of approximately $33 \mathrm{kcal} \mathrm{mol}^{-1}$, whereas this value is considerably decreased to approximately $17 \mathrm{kcal} \mathrm{mol}^{-1}$ for the case of meta-substitutions. ${ }^{23}$ Hexaphenylbenzene $\mathrm{C}_{6} \mathrm{Ph}_{6}$ consequently displays one set of signals in the ${ }^{13} \mathrm{C}$ NMR spectra and one overlapped signal with a center of gravity at $6.83 \mathrm{ppm}(30 \mathrm{H})$ in the proton resonance spectra. ${ }^{35}$ The chemical shifts of 10a-c are different as a consequence of their isomerism and, in addition, intermolecular interactions (Fig. 1). The broadening of the signals at approximately $7.00 \mathrm{ppm}$ in the spectrum of 10a (blue) can be attributed to $\mathrm{N} \cdots \mathrm{H}-\mathrm{C}$ interactions to the nitrogen atom, which is not possible in $\mathbf{1 0 b}, \mathbf{c}$. These interactions are thought to be within the limits of hydrogen bonds and classical van der Waals contacts. ${ }^{36}$ The upfield shift of phenyl ring signals due to intramolecular $\pi$-interactions caused by the quinolin-4-yl substitution of 10c can clearly be seen (black), which are less in 10a,b.

The steric hindrance of the quinoline rings can be compared with 1-(3,4-dimethylphenyl)-2,3,4,5,6-pentaphenylbenzene $^{37}$ for 10a,b and 1-(2,3-dimethylphenyl)-2,3,4,5,6-pentaphenylbenzene, which seems to be unknown, for 10c.
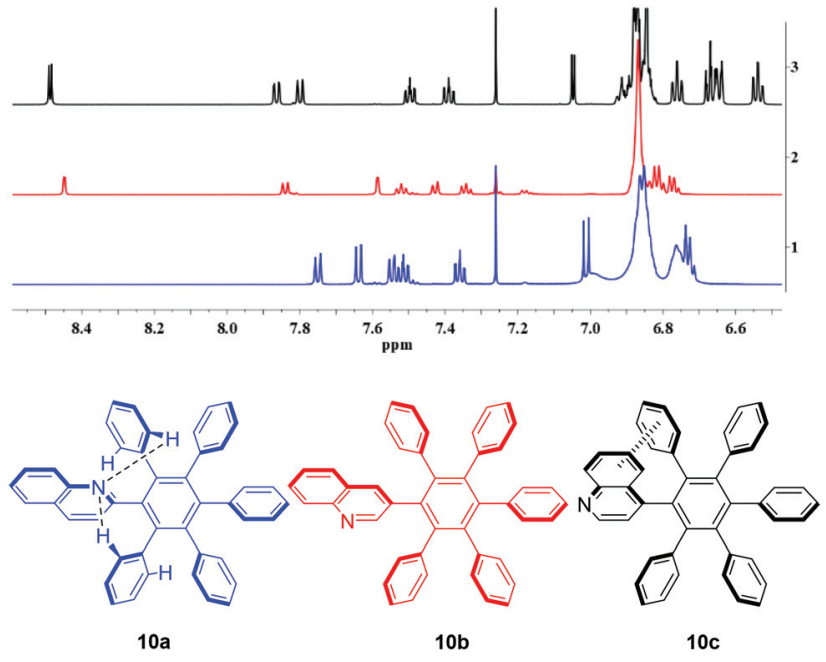

Fig. 1 Comparison of ${ }^{1} \mathrm{H}$ NMR spectra of HHABs 10a (1), 10b (2), and $10 \mathrm{c}(3)\left(\mathrm{DMSO}-\mathrm{d}_{6}, 25^{\circ} \mathrm{C}\right)$. 
However, the quinolin-4-yl-substitution pattern of 10c imitates the steric hindrance of the methyl group in the model compound 1-(2-methylphenyl)-2,3,4,5,6-pentaphenylbenzene which has a propeller-type topology in the $\operatorname{solid}^{38}$ and a slow rotation of the $o$-tolyl group $25{ }^{\circ} \mathrm{C}$ in solution. ${ }^{39}$ For the case of free rotation under the measurement conditions, 25 distinct ${ }^{13} \mathrm{C}$ NMR resonance frequencies can be expected for $10 a-c$, among those 15 signals of $\mathrm{CH}$ groups. For the case of restricted rotation, this number is increased to $29{ }^{13} \mathrm{C}$ NMR signals in total, and 19 signals of $\mathrm{CH}$ groups, because the ortho- and meta-positions of the 2- and 3-phenyl substituents become non-isochronous. The least sterically hindered compound 10a displays 22 distinct signals due to overlapping in $\mathrm{DMSO}^{-\mathrm{d}_{6}}$ at $25{ }^{\circ} \mathrm{C}$. Compound 10b shows the expected 29 signals for a restricted rotation, and 10c displays in total 27 distinct resonance frequencies under the same measurement conditions, presumably due to overlapping of two signals.

The same protocol was applied to the reaction of quinoline 14 with two alkynyl residues which was prepared in $21 \%$ yield in three steps from 1,4-dibromobenzene $\mathbf{1 1}$ via 12 and 13 as shown (Scheme 3). Quinoline 14 reacted with an excess of cyclopentadienone $\mathbf{9}$ to give compound $\mathbf{1 5}$ which precipitated as exclusive reaction product during the work-up procedure in good yield.

The method is also applicable to prepare isomeric diquinolinyl substituted tetraphenylbenzenes (Scheme 4). Thus, under analogous conditions, the compound 19a with quinolin-3-yl/ 3-yl connectivity and the isomeric quinolin-3-yl/4-yl derivative 19b were prepared in $90 \%$ and $78 \%$ yield, respectively, starting from 18a,b which are available starting from $\mathbf{6 b , c}$ and 17 by standard procedures. The quinolin-3-yl,3-yl compound 19a can exist in two diastereomeric forms due to restricted rotation, a $C_{\mathrm{s}}$ conformation and a racemic pair with $C_{2}$ symmetry, similar to hexaarylbenzenes with two meta-substituents at $0{ }^{\circ} \mathrm{C}$ on the NMR time scale. ${ }^{34,40}$ The ${ }^{1} \mathrm{H}$ NMR spectrum of 19a showed 37 of expectable $48{ }^{13} \mathrm{C}$ NMR signals. Two sets of partially overlapped signals can be identified in a ratio of $1: 0.8$ under the

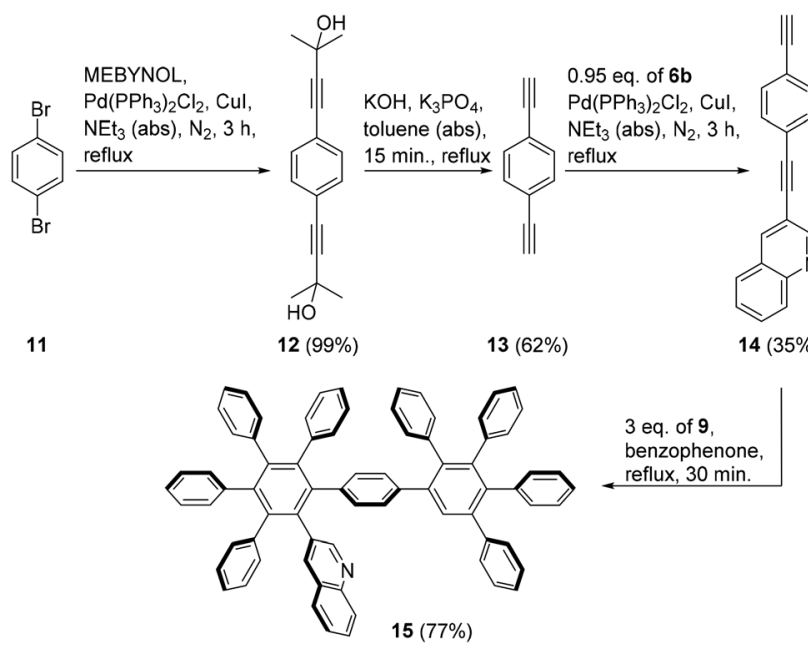

Scheme 3 Synthesis of a bulky monoquinoline HHAB.

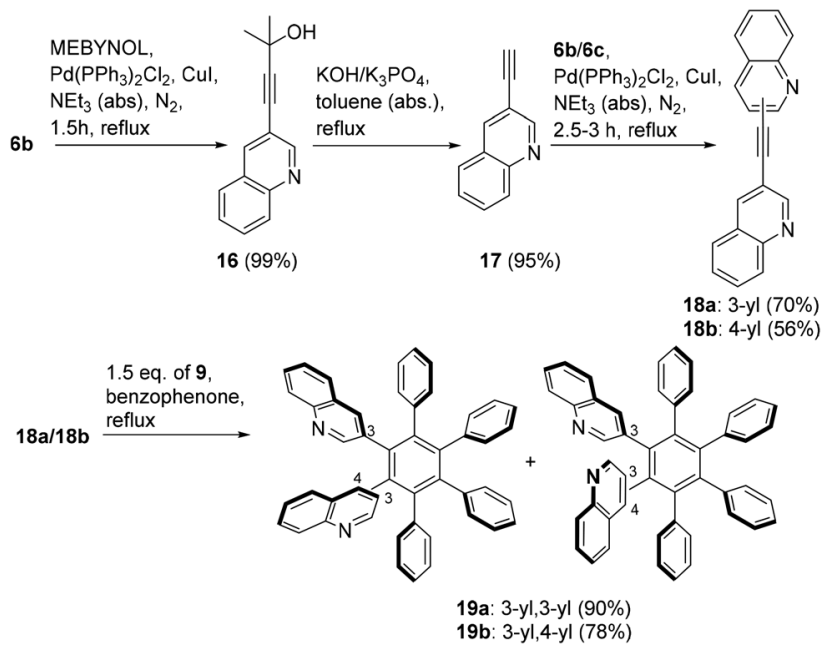

Scheme 4 Synthesis of diquinoline-substituted HHABs.

measuring conditions (DMSO- $d_{6}, 25{ }^{\circ} \mathrm{C}$ ). The 4-H hydrogen atom of the quinoline residues of one isomer appears at $7.97 \mathrm{ppm}$, whereas the other is detectable at $7.93 \mathrm{ppm}$. The ${ }^{13} \mathrm{C}$ NMR spectrum of isomer $19 \mathrm{~b}$, which possesses a quinolin3-yl,4-yl connectivity, shows 72 distinct signals of expectable 96. This number is due to the fact, that the 3-yl,4-yl connectivity causes an additional non-symmetry of the molecule and consequently non-isochronous substituents of the two magnetically inequivalent isomers. Under the same measuring conditions, two isochronous rotameric forms are present in a ratio of $1: 1$ in the NMR spectrum of $\mathbf{1 9 b}$.

For the synthesis of HHABs with three quinoline residues another approach was applied. Thus, the asymmetric acetylene 8a was subjected to a cobalt-catalyzed $[2+2+2]$-trimerization reaction which has already been applied for the synthesis of HABs before (Scheme 5). ${ }^{41}$ The trimerization of $8 \mathbf{a}$ gave a mixture of two separable regioisomers 20a and 20 b (1:3) with a total yield of $40 \%$. Compound 20 a is a heteroaromatic analogue of 1,3,5-tri( $\alpha$-naphthyl)-benzene, a propeller-like, nonplanar molecule that is known to interlock in the melt. ${ }^{42}$ It displays $15{ }^{13} \mathrm{C}$ NMR signals, similar to the corresponding number of signals of $D_{3 \mathrm{~h}}$ symmetric oligophenylenes which were expected within the fast exchange limit for all single bond rotations. ${ }^{43}$ Similar to the spectra of the mono(quinolin2-yl)derivative 10a, the signals of the ortho- and meta-protons

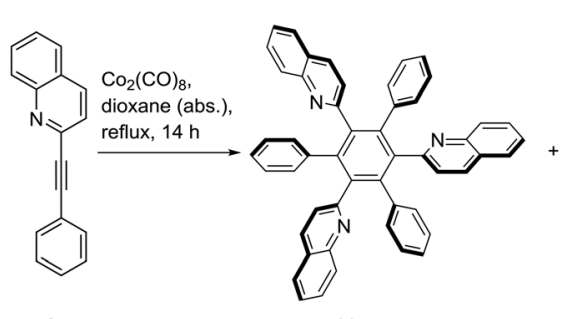

$20 a(10 \%)$

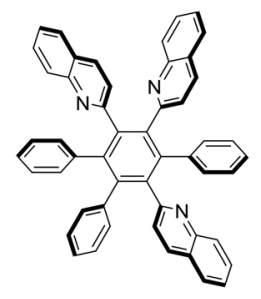

$20 \mathrm{~b}(30 \%)$
Scheme 5 Cobalt-catalyzed $[2+2+2]$-cyclotrimerization. 
appear as broad singlets due to $\mathrm{N} \cdots \mathrm{H}-\mathrm{C}$ interactions. Assignment of all atoms using 2D spectra was possible; one set of proton signals for all three quinoline residues of 20a was found. It is known that ortho-substitutions, which cannot be taken as a model for the quinolin-2-yl substitution, can prevent rapid interconversion of the $C_{3 \mathrm{v}}$ and $C_{\mathrm{s}}$ isomeric forms which are the result of the formation of $s y n$ - and anti-conformations. ${ }^{44}$ The 1,2,4-substituted compound $20 \mathrm{~b}$ displays 36 of expectable 45 distinct ${ }^{13} \mathrm{C}$ NMR resonance frequencies in DMSO- $\mathrm{d}_{6}$ at $25^{\circ} \mathrm{C}$, if free rotation under the measurement conditions is presumed. Similar to compound 10a, a considerable broadening of the ${ }^{1} \mathrm{H}$ NMR signals of the quinolines in DMSO$\mathrm{d}_{6}$ at $25^{\circ} \mathrm{C}$ as well as the phenyl rings is observable.

Trimerization of the acetylene 18a was tested to prepare a hexaquinolin-3-yl substituted benzene. Indeed, HHAB 21 was formed in 85\% yield (Scheme 6). Unfortunately, due to the rather limited solubility of $\mathbf{2 1}$ NMR analyses were not possible. Nonetheless, the corresponding HRMS $(m / z=863.2896)$ is in accord with the structure of $\mathbf{2 1}$ which can exist in eight rotameric forms. Literature-known ${ }^{23}$ calculated minimized energies of hexa( $\beta$-naphthyl)benzene, the non-heteroaromatic analogue of 21, revealed that the most stable rotamer is the one in which all $\beta$-naphthyl residues are twisted onto the same side $(6,0)$. The X-ray crystal analysis of hexa(2-pyridyl)benzene, however, revealed the $\alpha, \beta, \alpha, \beta, \alpha, \beta$ arrangement of pyridine rings and dihedral angles between the pyridyl substituent and the benzene ring of approximately $90^{\circ} .^{22}$

\section{Synthesis of polycationic HABs}

Based on former experiences of our group, ${ }^{45}$ dimethyl sulfate was used as a methylation agent to convert the quinoline derivatives into cationic species. First, the monoquinoline substituted HABs 10a-c were successfully methylated in anhydrous toluene under reflux conditions (Scheme 7). The $N$-methyl group of 22a interlocks the molecule and 19 distinct ${ }^{13} \mathrm{C}$ NMR signals of $\mathrm{CH}$ groups are detectable plus the signals of the anion and the methyl group at 52.8 and $42.5 \mathrm{ppm}$, respectively. Thus, in contrast to $10 \mathbf{a}-\mathbf{c}, \mathbf{2 2 a}-\mathbf{c}$ all display the same number of signals. The $N$-methylquinolinium salts of $\mathbf{1 5}$ with methylsulfate (23) and hexafluorophosphate anions $\left(\mathbf{2 3 P F} \mathbf{F}_{\mathbf{6}}\right)$ were prepared as well, and the diquinoline HABs 19a,b were also successfully methylated. An immediate anion exchange reaction during work-up yielded the dihexafluoro-
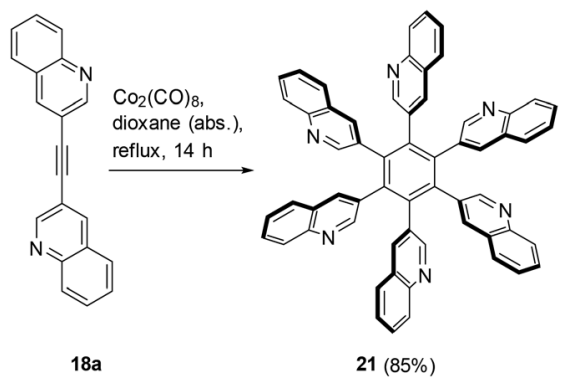

Scheme 6 Cobalt-catalyzed $[2+2+2]$-cyclotrimerization of 18 a.
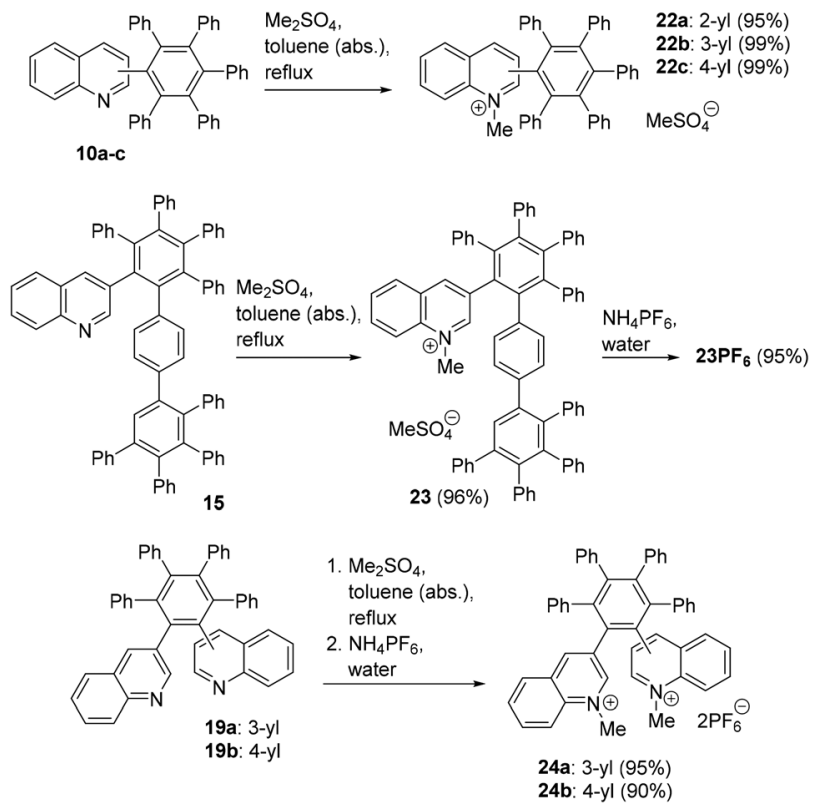

Scheme 7 Methylation of quinoline-based HHABs.

phosphate salts $\mathbf{2 4 a}, \mathbf{b}$. Similar to the non-methylated precursors $19 \mathrm{a}$ and $19 \mathrm{~b}$, the number of ${ }^{13} \mathrm{C}$ NMR resonance frequencies of $\mathbf{2 4 a}$ and $\mathbf{2 4 b}$ is 38 and 72, respectively. The 3-yl,3-yl salt 24a has an almost identical ratio of rotamers $(1: 0.9)$ as its non-methylated precursor, and the corresponding ratio of the salt 24b in DMSO- $d_{6}$ is $1: 1.27$. In summary, except for 10a, no spectroscopically detectable changes of the symmetry of the molecules is caused by the methylations. On methylation, the UV/Vis absorption maxima display bathochromic shifts. The spectra are shown in the ESI. $\dagger$

Finally, $\mathrm{N}$-methylation of $\mathbf{2 1}$ with an excess of dimethyl sulfate followed by precipitation with $\mathrm{NH}_{4} \mathrm{PF}_{6}$ successfully gave the fully methylated hexacationic HHAB 25 (Scheme 8) which is soluble in DMSO- $\mathrm{d}_{6}$ and which could be characterized completely.

Theoretically, the salt 25 can exist in eight rotameric forms A-H. Similar to hexa( $\beta$-naphthyl)benzene, ${ }^{23}$ solving the simple combinatoric problem created by propeller-like compounds such as 21 or 25 results in the following ratio: $A: B: C: D: E: F: G: H=1: 6: 3: 6: 6: 3: 6: 1$. Fig. 2 shows

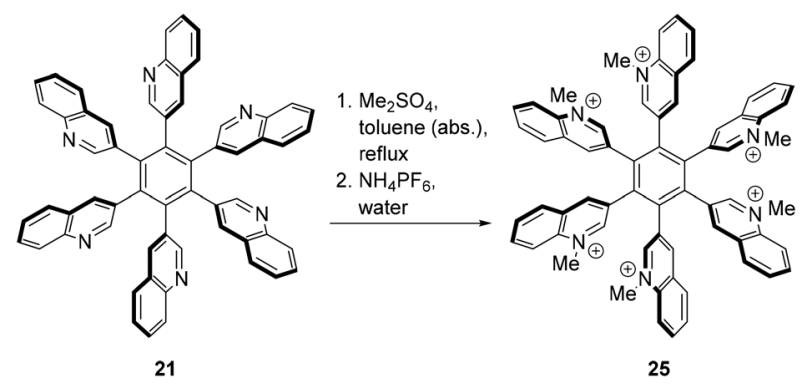

Scheme 8 Methylation of perquinoline-substituted HAB 21. 


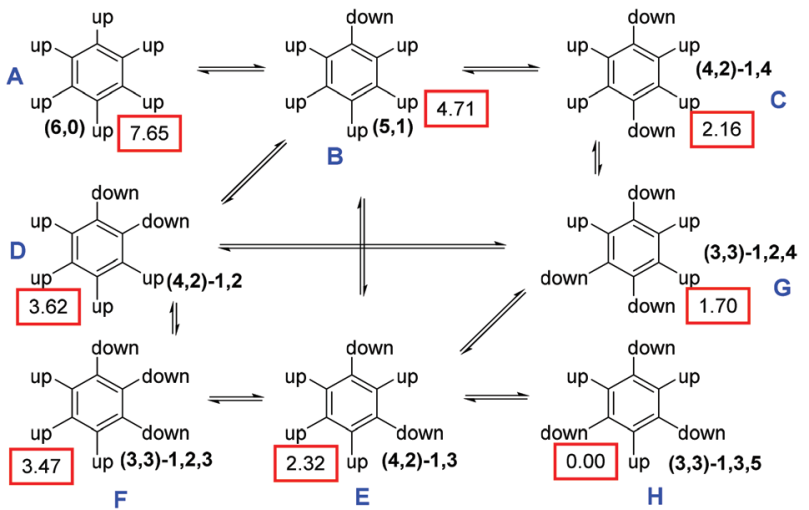

Fig. 2 Eight energy-minimized rotamers of 25, showing which can be directly interconverted by rotation of a single substituent. Relative energies (in $\mathrm{kJ} \mathrm{mol}^{-1}$ ) are shown in red boxes.

possible interconversions between the forms $\mathrm{A}-\mathrm{H}$, which we calculated in form of the hexacationic species (DFT, 6-31G*) PBE0). Schematically, the rotamers can be classified as those in which $N$-methylquinolinium residues are $6: 0$ (all-syn), 5-up/ 1-down, 4 up/2-down $(1,2 ; 1,3 ; 1,4)$, and 3-up/3-down $(1,2,3$; $1,2,4 ; 1,3,5)$ (Fig. 2). The calculated energy differences between the eight rotamers of $\mathbf{2 5}$ are extremely small, and so are the differences between the rotamers of 21. The lowest energy rotamer of 25 is the 3-up-3-down $(1,3,5)$ isomer, whereas the all-syn isomer $(6,0)$ has an energy of $7.65 \mathrm{~kJ} \mathrm{~mol}^{-1}$ and is thus the less stable. Similarly, the $(6,0)$ rotamer of the neutral precursor 21 is by only $3.8 \mathrm{~kJ} \mathrm{~mol}^{-1}$ less stable than the $(3,3)-1,3,5$ rotamer. As a matter of fact, the rotamers of 25 cause signal overlaps in the ${ }^{1} \mathrm{H}$ NMR spectrum at ambient temperature (Fig. 1, spectrum 1, ESI $\dagger$ ). The spectra change reversibly on heating of the NMR sample successively from rt to $100{ }^{\circ} \mathrm{C}$ (Fig. 1, spectra 2-9); on cooling, the original spectrum is reconstituted (Fig. 1, spectrum 10). Obviously, no other ratio of sets of isochronous rotamers is formed during this temperature experiment. Calculations of all true minimum structures of $\mathbf{2 5}$ show that in all rotamers the dihedral angles between the quinoline and the phenyl rings are in the range from $71^{\circ}$ to $88^{\circ}$.

Fig. 3 shows the calculated most stable conformer of 25 and the HOMO/LUMO profiles of the $25(3,3)-1,3,5$ rotamer. In contrast to $\mathbf{2 2 b},^{46}$ in which the HOMO is separated from the LUMO, the HOMO $(-19.41 \mathrm{eV})$ as well as LUMO $(-15.48 \mathrm{eV})$ of 25 are located individually on the six $N$-methylquinolinium-3yl residues. Considerable parts of the LUMO are located on the nitrogen atoms as well as on the $\mathrm{C} 2 / \mathrm{C} 4$ carbon atoms of the quinolinium residues, whereas significant parts of the HOMO are located on their fused benzene rings. The fraction of the HOMO located at C3 of the (3,3)-1,3,5- as well as of the all-syn $(6,0)$ rotamer $(c f$. ESI $\dagger)$ is lying in one plane and is orientated to the opposite phases of neighboring $\mathrm{C} 3$, similar to the inplane-aromatic stabilizations due to interactions between the in-plane ethynyl $\pi$-orbitals in hexaethynylbenzene. ${ }^{47}$ This may contribute to the stability of 25 . The frontier orbitals of the neutral precursor $\mathbf{2 1}$ are shown in the ESI. $\dagger$
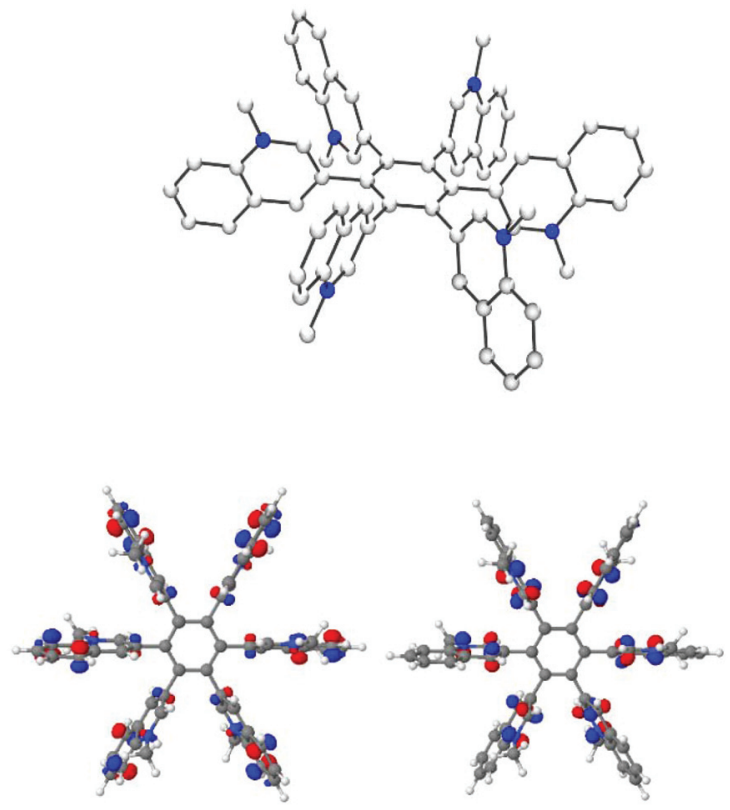

Fig. 3 Most stable conformation of 25 (calculated) (above). HOMO (left) and LUMO (right) of $25(3,3)-1,3,5$.

\section{Conclusions}

Series of propeller-shaped quinolinium-substituted benzenes as well as their electrostatically neutral precursors have been prepared under variation of the substitution site of the quinoline ring (2-yl, 3-yl, 4-yl), covering the range from the monocationic quinolinium-2,3,4,5,6-pentaphenylbenzene to the hexacationic hexakis(1-methylquinolinium-3-yl)benzene. The latter can exist in eight different rotamers, the $(3,3)-1,3,5$-isomer of which was calculated to be the most stable one. Due to the propeller-shape geometry which suppresses conjugation throughout the entire $\pi$-electron system, the frontier orbitals are located in the individual quinolinium rings.

\section{Experimental}

All reactions were carried out under an atmosphere of nitrogen in flame or oven-dried glassware. All chemicals were purchased and used without further purification unless otherwise mentioned. Anhydrous solvents were dried according to standard procedures before usage. Melting points are uncorrected and were determined in an apparatus according to Dr Tottoli (Büchi). The ATR-IR spectra were obtained on a Bruker Alpha in the range of 400 to $4000 \mathrm{~cm}^{-1}$. ${ }^{1} \mathrm{H}$ NMR spectra were recorded at $600 \mathrm{MHz} .{ }^{13} \mathrm{C} \mathrm{NMR}$ spectra were recorded at $150 \mathrm{MHz}$, with the solvent peak used as the internal reference. Multiplicities are described by using the following abbreviations: $\mathrm{s}=$ singlet, $\mathrm{d}=$ doublet, $\mathrm{t}=$ triplet, $\mathrm{q}=$ quartet, and $\mathrm{m}=$ multiplet. Spectroscopic atom numberings are shown in the ESI. $\dagger$ Signal orientations in DEPT experiments were described as follows: o = no signal; + = up $\left(\mathrm{CH}, \mathrm{CH}_{3}\right) ;-=\operatorname{down}\left(\mathrm{CH}_{2}\right)$. 
The mass spectra (ESIMS) were measured with a Varian 320 MS Triple Quad GC/MS/MS (EIMS) or with an Agilent LCMSD series HP 1100 with APIES at fragmentor voltages as indicated. Samples were sprayed from $\mathrm{MeOH}$ at $4000 \mathrm{~V}$ capillary voltage and fragmentor voltages of $30 \mathrm{~V}$ unless otherwise noted. The HRMS spectra were obtained with a Bruker Impact II, a Bruker Daltonik Tesla-Fourier transform-ion cyclotron resonance mass spectrometer, or with a Waters Micromass LCT with the direct inlet. Chromatography: The reactions were traced by thin layer chromatography with silica gel 60 (F254, company MERCK KGAA). For the detection of substances, quenching was used at either $254 \mathrm{~nm}$ or $366 \mathrm{~nm}$ with a mercury lamp. The preparative column chromatography was conducted through silica gel 60 (230-400 mesh) of the company MERCK KGAA. Yields are not optimized.

\section{Calculations}

All density-functional theory (DFT)-calculations were carried out by using the Firefly 8.2.0 QC package, ${ }^{48}$ which is partially based on the GAMESS (US) source code, ${ }^{49}$ running on Linux 2.6.18-238.el5 SMP (x86_64) on five AMD Phenom II X6 1090T processor workstations (Beowulf-cluster) with Infiniband interconnect and parallelized with MPICH 1.2.7p1. MM2 optimized structures were used as starting geometries. Complete geometry optimizations were carried out on the implemented $6-31 \mathrm{G}^{*}$ basis set and with the PBE0 density functional. All calculated structures were proven to be true minima by the absence of imaginary frequencies. Partial charges were obtained with NBO $5.9^{50}$ from the results of the DFT calculations. Orbital plots were obtained using Jmol 14.27.2.

\section{Synthesis}

\section{General procedure of Sonogashira-Hagihara coupling (Procedure 1)}

The reactions were carried out under a nitrogen atmosphere. A mixture of $5 \mathrm{mmol}$ of corresponding haloquinoline 1, $1 \mathrm{~mol} \%$ of $\mathrm{Pd}\left(\mathrm{PPh}_{3}\right)_{2} \mathrm{Cl}_{2}$, and $2 \mathrm{~mol} \%$ of CuI was suspended in $7 \mathrm{~mL}$ of dry $\mathrm{NEt}_{3}$ with stirring. A sample of the corresponding ethynylbenzene 2 (1.05 equiv.) in dry $\mathrm{NEt}_{3}$ was added dropwise at ambient temperature. The resulting solutions were then stirred at reflux temperature until complete conversion was monitored by TLC. The mixtures were then allowed to cool to rt. The solvents were removed in vacuo. The resulting residues were finally purified by column chromatography (petroleum ether : ethyl acetate) to afford the products.

2-(Phenylethynyl)quinoline (8a). According to Procedure 1 , a solution of $0.491 \mathrm{~g}(3.00 \mathrm{mmol})$ of 2-chloroquinoline $6 \mathbf{a}$, $0.042 \mathrm{~g}(0.06 \mathrm{mmol})$ of $\mathrm{Pd}\left(\mathrm{PPh}_{3}\right)_{2} \mathrm{Cl}_{2}, 0.012 \mathrm{~g}(0.06 \mathrm{mmol}) \mathrm{CuI}$ and $0.367 \mathrm{~g}(3.6 \mathrm{mmol})$ of ethynylbenzene $7 \mathrm{in} 20 \mathrm{~mL}$ of anhydrous $\mathrm{NEt}_{3}$ was heated over the period of $2 \mathrm{~h}$ under reflux temperature. Finally, a purification by column chromatography (petroleum ether: ethyl acetate $=5: 1$ ) gave 2 -(phenylethynyl) quinoline 8a. Yield $0.653 \mathrm{~g}, 95 \%$, a yellow solid, m.p. $75^{\circ} \mathrm{C}$ (72-75 ${ }^{\circ} \mathrm{C}$ (ref. 51)). ${ }^{1} \mathrm{H}$ NMR $\left(600 \mathrm{MHz}, \mathrm{CDCl}_{3}\right): \delta=8.44(\mathrm{~d}, J=$
$8.3 \mathrm{~Hz}, 1 \mathrm{H}, 4-\mathrm{H}), 8.03-8.01(\mathrm{~m}, 2 \mathrm{H}, 5-\mathrm{H}, 8-\mathrm{H}), 7.82$ (ddd, $J=$ 1.5, 6.7, $8.5 \mathrm{~Hz}, 1 \mathrm{H}, 7-\mathrm{H}), 7.74$ (d, $J=8.3 \mathrm{~Hz}, 1 \mathrm{H}, 3-\mathrm{H})$, $7.70-7.68$ (m, 2H, 2'-H, 6'-H), 7.66 (ddd, $J=1.2,6.9,8.1 \mathrm{~Hz}, 1 \mathrm{H}$, 6-H), 7.53-7.48 (m, 3H, 3'-H, 4'-H, 5'-H) ppm. ${ }^{13} \mathrm{C}$ NMR (150 MHz, $\mathrm{CDCl}_{3}$ ): $\delta=147.7$ (o, C8a), 142.6 (o, C2), 136.7 (+, C4), 131.9 (+, C2', C6'), 129.7 (+, C4'), 128.9 (+, C3', C5'), 128.6 (+, C5), 128.0 (+, C8), 127.4 (+, C6), 126.9 (o, C4a), 124.3 (+, C3), 121.2 (o, C1'), 89.5 (o, C $\alpha$ ), 89.1 (o, C $\beta$ ) ppm. IR (ATR): 3053, 2208, 2161, 1616, 1591, 1552, 1498, 1441, 1425, 1310, 1295, 1211, 1142, 1115, 1070, 989, 829, 788, 769, 760, 693, 620, 548, 526, $478 \mathrm{~cm}^{-1}$. HRMS (ESI): $\mathrm{m} / z$ calcd for $\mathrm{C}_{17} \mathrm{H}_{12} \mathrm{~N}[\mathrm{M}+$ $\mathrm{H}]^{+}$230.0965, found 230.0969; $\mathrm{m} / z$ calcd for $\mathrm{C}_{17} \mathrm{H}_{11} \mathrm{NNa}[\mathrm{M}+$ $\mathrm{Na}]^{+}$252.0789, found 252.0789.

3-(Phenylethynyl)quinoline (8b). According to Procedure 1, a solution of $2.080 \mathrm{~g}(10.00 \mathrm{mmol})$ of 3 -bromoquinoline $\mathbf{6 b}$, $0.070 \mathrm{~g}(0.10 \mathrm{mmol})$ of $\mathrm{Pd}\left(\mathrm{PPh}_{3}\right)_{2} \mathrm{Cl}_{2}, 0.038 \mathrm{~g}(0.20 \mathrm{mmol}) \mathrm{CuI}$ and $1.071 \mathrm{~g}(10.50 \mathrm{mmol})$ of ethynylbenzene 7 in $30 \mathrm{~mL}$ of anhydrous $\mathrm{NEt}_{3}$ was heated over the period of $1.5 \mathrm{~h}$ under reflux temperature. Finally, a purification by column chromatography (petroleum ether: ethyl acetate $=3: 1$ ) gave 3-(phenyl-ethynyl)quinoline 8b. Yield $2.290 \mathrm{~g}, 100 \%$, a white solid, m.p. $83{ }^{\circ} \mathrm{C} .{ }^{1} \mathrm{H}$ NMR $\left(600 \mathrm{MHz}, \mathrm{CDCl}_{3}\right): \delta=9.00(\mathrm{~d}, J=$ $2.0 \mathrm{~Hz}, 1 \mathrm{H}, 2-\mathrm{H}), 8.30(\mathrm{~d}, J=2.0 \mathrm{~Hz}, 1 \mathrm{H}, 4-\mathrm{H}), 8.10(\mathrm{~d}, J=$ $8.3 \mathrm{~Hz}, 1 \mathrm{H}, 8-\mathrm{H}), 7.79(\mathrm{~d}, J=8.3 \mathrm{~Hz}, 1 \mathrm{H}, 5-\mathrm{H}), 7.72$ (ddd, $J=$ 1.5, 6.9, 8.3 Hz, 1H, 7-H), 7.63-7.53 (m, 3H, 6-H, 2'- $\left.\mathrm{H}, 6^{\prime}-\mathrm{H}\right)$, 7.42-7.35 (m, 3H, 3'-H, 4'-H, 5'-H) ppm. ${ }^{13} \mathrm{C}$ NMR $(150 \mathrm{MHz}$, $\left.\mathrm{CDCl}_{3}\right): \delta=152.1(+, \mathrm{C} 2), 146.8(\mathrm{o}, \mathrm{C} 8 \mathrm{a}), 138.3(+, \mathrm{C} 4), 131.8(+$, C2', C6'), 130.1 (+, C7), $129.4(+, \mathrm{C} 8), 128.9\left(+, \mathrm{C} 4{ }^{\prime}\right), 128.5(+$, C3', C5'), 127.6 (+, C6), 127.3 (+, C5), 127.3 (o, C4a), 122.6 (o, C1') 117.5 (o, C3), 92.7 (o, C $\beta$ ), 86.7 (o, C $\alpha$ ) ppm. IR (ATR): 3008, 2162, 1619, 1599, 1564, 1484, 1443, 1409, 1368, 1294, 1196, 1124, 1113, 1072, 980, 905, 860, 784, 756, 691, 656, 640, 539, 515, 498, 474, $429 \mathrm{~cm}^{-1}$. HRMS (ESI): $\mathrm{m} / z$ calcd for $\mathrm{C}_{17} \mathrm{H}_{11} \mathrm{NNa}[\mathrm{M}+\mathrm{Na}]^{+}$252.0789, found 252.0796.

4-(Phenylethynyl)quinoline (8c). According to Procedure 1 , a solution of $0.624 \mathrm{~g}(3.00 \mathrm{mmol})$ of 4 -bromoquinoline $6 \mathrm{c}$, $0.042 \mathrm{~g}(0.06 \mathrm{mmol})$ of $\mathrm{Pd}\left(\mathrm{PPh}_{3}\right)_{2} \mathrm{Cl}_{2}, 0.012 \mathrm{~g}(0.06 \mathrm{mmol}) \mathrm{CuI}$ and $0.367 \mathrm{~g}$ ( $3.6 \mathrm{mmol})$ of ethynylbenzene 7 in $20 \mathrm{~mL}$ of anhydrous $\mathrm{NEt}_{3}$ was heated over the period of $2 \mathrm{~h}$ under reflux temperature. Finally, a purification by column chromatography (petroleum ether: ethyl acetate $=5: 1$ ) gave 4-(phenylethynyl) quinoline 8c. Yield $0.674 \mathrm{~g}, 98 \%$, of a yellow solid, m.p. $45^{\circ} \mathrm{C}$. ${ }^{1} \mathrm{H}$ NMR (600 MHz, $\mathrm{CDCl}_{3}$ ): $\delta=8.90(\mathrm{~d}, J=4.4 \mathrm{~Hz}, 1 \mathrm{H}, 2-\mathrm{H})$, 8.38 (ddd, $J=0.6,1.4,8.3 \mathrm{~Hz}, 1 \mathrm{H}, 5-\mathrm{H}), 8.14$ (d, $J=8.1 \mathrm{~Hz}, 1 \mathrm{H}$, $8-\mathrm{H}), 7.76$ (ddd, $J=1.4,6.9,8.4 \mathrm{~Hz}, 1 \mathrm{H}, 7-\mathrm{H}), 7.68-7.66(\mathrm{~m}, 2 \mathrm{H}$, 2'-H, 6'-H), 7.64 (ddd, $J=1.4,6.9,8.2 \mathrm{~Hz}, 1 \mathrm{H}, 6-\mathrm{H}), 7.57$ (d, $J=$ $4.4 \mathrm{~Hz}, 1 \mathrm{H}, 3-\mathrm{H}), 7.44-7.42$ (m, 3H, 3'-H, 4'-H, 5'-H) ppm. ${ }^{13} \mathrm{C}$ NMR (150 MHz, $\mathrm{CDCl}_{3}$ ): $\delta=149.9$ (,$\left.+ \mathrm{C} 2\right), 148.3$ (o, C8a), 132.1 (+, C2', C6'), 130.0 (+, C7, C8), 129.9 (o, C4), 129.5 (+, C4'), 128.7 (+, C3', C5'), 127.9 (o, C4a), 127.3 (+, C6), 126.1 (+, C5), 123.7 (+, C3), 122.4 (o, C1'), 98.8 (o, C $\beta), 85.2$ (o, C $\alpha$ ) ppm. IR (ATR): 3085, 3051, 3029, 2998, 2212, 1961, 1919, 1595, 1576, $1505,1487,1441,1418,1390,1363,1310,1277,1217,1192$, 1175, 1134, 1068, 1029, 919, 871, 846, 815, 756, 687, 641, 579, 550, 529, 506, 485, $442 \mathrm{~cm}^{-1}$. HRMS (ESI): $\mathrm{m} / \mathrm{z}$ calcd for $\mathrm{C}_{17} \mathrm{H}_{12} \mathrm{~N}[\mathrm{M}+\mathrm{H}]^{+}$230.0965, found 230.0965. 
General procedure of preparation of propeller-like compounds (Procedure 2)

Benzophenone $(10 \mathrm{~g})$ was melted in a $50 \mathrm{~mL}$ round-bottomed flask fitted with an air condenser. Corresponding phenylethynylquinoline $8(2.00 \mathrm{mmol})$ and tetraphenylcyclopentadienone $9(2.50 \mathrm{mmol})$ were added to the flask, which was heated for $0.5 \mathrm{~h}$ using a heat gun. The solution was cooled to rt and toluene $(10 \mathrm{~mL})$ was added to prevent the solidification of the benzophenone. After cooling, $n$-hexane $(50 \mathrm{~mL})$ was added, resulting in the precipitation of a product, which was collected by vacuum filtration.

\section{2,3,4,5,6-Pentaphenyl-1-(quinolin-2-yl)benzene}

(10a). 2,3,4,5,6-Pentaphenyl-1-(quinoline-2-yl)benzene 10a was prepared by Procedure 2 using 2-(phenylethynyl)quinoline 8a (0.158 g, $0.690 \mathrm{mmol})$ and tetraphenylcyclopentadienone 9 $(0.394 \mathrm{~g}, 1.035 \mathrm{mmol})$ in benzophenone $(5 \mathrm{~g})$ in $10 \mathrm{~mL}$ roundbottomed flask in $0.75 \mathrm{~h}$. Yield $0.260 \mathrm{~g}, 64 \%$, a white solid, m.p. $>360{ }^{\circ} \mathrm{C} .{ }^{1} \mathrm{H}$ NMR $\left(600 \mathrm{MHz}, \mathrm{CDCl}_{3}\right): \delta=7.75(\mathrm{~d}, J=$ $\left.8.6 \mathrm{~Hz}, 1 \mathrm{H}, 8^{\mathrm{i}}-\mathrm{H}\right), 7.64\left(\mathrm{~d}, J=8.5 \mathrm{~Hz}, 1 \mathrm{H}, 4^{\mathrm{i}}-\mathrm{H}\right), 7.55(\mathrm{dd}, J=1.2$, $8.2 \mathrm{~Hz}, 1 \mathrm{H}, 5^{\mathrm{i}}-\mathrm{H}$ ), 7.51 (ddd, $\left.J=1.5,6.9,8.5 \mathrm{~Hz}, 1 \mathrm{H}, 7^{\mathrm{i}}-\mathrm{H}\right), 7.36$ (ddd, $\left.J=1.1,7.0,8.1 \mathrm{~Hz}, 1 \mathrm{H}, 6^{\mathrm{i}}-\mathrm{H}\right), 7.01(\mathrm{~d}, J=8.5 \mathrm{~Hz}, 1 \mathrm{H}$, $\left.3^{\mathrm{i}}-\mathrm{H}\right), 7.00-6.96(\mathrm{~m}, 2 \mathrm{H}, \mathrm{Ph}), 6.93-6.83\left(\mathrm{~m}, 16 \mathrm{H}, 2^{\mathrm{ii}}-\mathrm{H}, 6^{\mathrm{ii}}-\mathrm{H}\right.$, $2^{\mathrm{iii}}-\mathrm{H}, 6^{\mathrm{iii}}-\mathrm{H}, 2^{\mathrm{v}}-\mathrm{H}, 6^{\mathrm{v}}-\mathrm{H}, 2^{\mathrm{vi}}-\mathrm{H}, 6^{\mathrm{vi}}-\mathrm{H}, 3^{\mathrm{ii}}-\mathrm{H}, 5^{\mathrm{ii}}-\mathrm{H}, 3^{\mathrm{iii}}-\mathrm{H}, 5^{\mathrm{iii}}-\mathrm{H}$, $\left.3^{\mathrm{v}}-\mathrm{H}, 5^{\mathrm{v}}-\mathrm{H}, 3^{\mathrm{vi}}-\mathrm{H}, 5^{\mathrm{vi}}-\mathrm{H}\right), 6.79-6.72(\mathrm{~m}, 7 \mathrm{H}, \mathrm{Ph}) \mathrm{ppm} .{ }^{13} \mathrm{C}$ NMR $\left(150 \mathrm{MHz}, \mathrm{CDCl}_{3}\right): \delta=160.2\left(\mathrm{o}, \mathrm{C} 2{ }^{\mathrm{i}}\right), 147.1\left(\mathrm{o}, \mathrm{C} 8 \mathrm{a}^{\mathrm{i}}\right), 141.6$ (o, $\mathrm{C}^{\text {iv }}$ ), 140.8/140.4/140.3/140.1 (o, C2, C3, C5, C6, C1 ${ }^{\mathrm{ii}}, \mathrm{C} 1^{\mathrm{iii}}$, $\mathrm{C}^{\mathrm{v}}, \mathrm{C} 1^{\mathrm{vi}}$ ), 140.6 (o, C4), 140.0 (o, C1), $134.2\left(+, \mathrm{C} 4^{\mathrm{i}}\right), 131.7 /$ 131.5/131.4/130.7 (+, Ph), $129.05\left(+, \mathrm{C}^{\mathrm{i}}\right), 129.00\left(+, \mathrm{C}^{\mathrm{i}}\right), 127.3$ $\left(+, \mathrm{C}^{\mathrm{i}}\right), 126.8(+, \mathrm{Ph}), 126.0\left(+, \mathrm{C6}^{\mathrm{i}}\right), 125.7\left(\mathrm{o}, \mathrm{C} 4 \mathrm{a}^{\mathrm{i}}\right), 125.51 /$ $125.45(+, \mathrm{Ph})$ ppm. IR (ATR): 3055, 3025, 1597, 1558, 1501, 1441, 1403, 1323, 1300, 1221, 1154, 1073, 1029, 948, 839, 812, 753, 718, 695, 617, 583, 555, 531, $478 \mathrm{~cm}^{-1}$. HRMS (ESI): $\mathrm{m} / z$ calcd for $\mathrm{C}_{45} \mathrm{H}_{32} \mathrm{~N}[\mathrm{M}+\mathrm{H}]^{+}$586.2530, found 586.2531.

2,3,4,5,6-Pentaphenyl-1-(quinolin-3-yl)benzene (10b). 2,3,4,5,6-Pentaphenyl-1-(quinoline-3-yl)benzene $\mathbf{1 0 b}$ was prepared by Procedure 2 using 3-(phenylethynyl)quinoline $\mathbf{8 b}$ (0.458 g, $2.00 \mathrm{mmol})$ and tetraphenylcyclopentadienone 9 $(0.961 \mathrm{~g}, 2.50 \mathrm{mmol})$ in benzophenone $(10 \mathrm{~g})$ in $50 \mathrm{~mL}$ roundbottomed flask in $0.5 \mathrm{~h}$. Yield $0.590 \mathrm{~g}, 51 \%$, a white solid, m.p. $334{ }^{\circ} \mathrm{C} .{ }^{1} \mathrm{H}$ NMR $\left(600 \mathrm{MHz}, \mathrm{CDCl}_{3}\right): \delta=8.45$ (d, $J=2 \mathrm{~Hz}$, $1 \mathrm{H}, 2 \mathrm{i}-\mathrm{H}), 7.84$ (d, $J=8.4 \mathrm{~Hz}, 1 \mathrm{H}, 8 \mathrm{i}-\mathrm{H}), 7.59$ (d, $J=2 \mathrm{~Hz}, 1 \mathrm{H}$, $4 \mathrm{i}-\mathrm{H}), 7.53-7.51$ (m, 1H, $\left.7^{\mathrm{i}}-\mathrm{H}\right), 7.43$ (d, $\left.J=8.0 \mathrm{~Hz}, 5^{\mathrm{i}}-\mathrm{H}\right)$, 7.35-7.33 (m, 1H, 6 $\left.6^{\mathrm{i}}-\mathrm{H}\right)$, 6.88-6.85 (m, 20H, $2^{\mathrm{ii}}-\mathrm{H}, 6^{\mathrm{ii}}-\mathrm{H}, 2^{\mathrm{iii}}-\mathrm{H}$, $6^{\mathrm{iii}}-\mathrm{H}, 2^{\mathrm{iv}}-\mathrm{H}, 6^{\mathrm{iv}}-\mathrm{H}, 2^{\mathrm{v}}-\mathrm{H}, 6^{\mathrm{v}}-\mathrm{H}, 2^{\mathrm{vi}}-\mathrm{H}, 6^{\mathrm{vi}}-\mathrm{H}, 3^{\mathrm{ii}}-\mathrm{H}, 5^{\mathrm{ii}}-\mathrm{H}, 3^{\mathrm{iii}}-\mathrm{H}$, $\left.5^{\mathrm{iii}}-\mathrm{H}, 3^{\mathrm{iv}}-\mathrm{H}, 5^{\mathrm{iv}}-\mathrm{H}, 3^{\mathrm{v}}-\mathrm{H}, 5^{\mathrm{v}}-\mathrm{H}, 3^{\mathrm{vi}}-\mathrm{H}, 5^{\mathrm{vi}}-\mathrm{H}\right), 6.83-6.78(\mathrm{~m}, 4 \mathrm{H}$, $\left.4^{\mathrm{ii}}-\mathrm{H}, 4^{\mathrm{iii}}-\mathrm{H}, 4^{\mathrm{v}}-\mathrm{H}, 4^{\mathrm{vi}}-\mathrm{H}\right), 6.78-6.77\left(\mathrm{~m}, 1 \mathrm{H}, 4^{\mathrm{iv}}-\mathrm{H}\right) \mathrm{ppm} .{ }^{13} \mathrm{C}$ NMR (150 MHz, $\left.\mathrm{CDCl}_{3}\right): \delta=152.9\left(+, \mathrm{C}^{\mathrm{i}}\right), 145.6\left(\mathrm{o}, \mathrm{C} 8 \mathrm{a}^{\mathrm{i}}\right)$, 141.4 (o, C4), 141.07 (o, C2, C6), 140.88 (o, C3, C5), 140.44 (o, $\left.\mathrm{C} 1^{\mathrm{iv}}\right), 140.34\left(\mathrm{o}, \mathrm{C} 1^{\mathrm{ii}}, \mathrm{C} 1^{\mathrm{vi}}\right), 139.96\left(\mathrm{o}, \mathrm{C} 1^{\mathrm{iii}}, \mathrm{C} 1^{\mathrm{v}}\right), 137.8\left(+, \mathrm{C} 4^{\mathrm{i}}\right)$, 136.51 (o, C1), 134.2 (o, C3 ${ }^{\mathrm{i}}$ ), 131.51/131.48/131.45/131.41/ $131.37\left(+, \mathrm{C} 2^{\mathrm{ii}}, \mathrm{C}^{\mathrm{ii}}, \mathrm{C} 2^{\mathrm{iii}}, \mathrm{C} 6^{\mathrm{iii}}, \mathrm{C} 2^{\mathrm{iv}}, \mathrm{C} 6^{\mathrm{iv}}, \mathrm{C} 2^{\mathrm{v}}, \mathrm{C}^{\mathrm{v}}, \mathrm{C} 2^{\mathrm{vi}}, \mathrm{C}^{\mathrm{vi}}\right)$, $129.0\left(+, \mathrm{C} 8^{\mathrm{i}}\right), 128.9\left(+, \mathrm{C} 7^{\mathrm{i}}\right), 127.6\left(+, \mathrm{C}^{\mathrm{i}}\right), 127.4 / 127.0 / 126.85 /$ $126.83 / 126.78\left(+, \mathrm{C} 3^{\mathrm{ii}}, \mathrm{C} 5^{\mathrm{ii}}, \mathrm{C} 3^{\mathrm{iii}}, \mathrm{C} 5^{\mathrm{iii}}, \mathrm{C} 3^{\mathrm{iv}}, \mathrm{C} 5^{\mathrm{iv}}, \mathrm{C} 3^{\mathrm{v}}, \mathrm{C} 5^{\mathrm{v}}, \mathrm{C} 3^{\mathrm{vi}}\right.$, $\left.\mathrm{C}^{\mathrm{vi}}\right), 127.1\left(\mathrm{o}, \mathrm{C} 4 \mathrm{a}^{\mathrm{i}}\right), 126.2\left(+, \mathrm{C}^{\mathrm{i}}\right), 125.87\left(+, \mathrm{C} 4^{\mathrm{ii}}, \mathrm{C} 4^{\mathrm{vi}}\right)$, $125.54\left(+, \mathrm{C}^{\mathrm{iii}}, \mathrm{C} 4^{\mathrm{v}}\right), 125.52\left(+, \mathrm{C} 4^{\mathrm{iv}}\right)$ ppm. IR (ATR): 3055,
3024, 1662, 1600, 1495, 1440, 1401, 1354, 1311, 1275, 1261, 1127, 1070, 1029, 966, 906, 838, 817, 779, 747, 738, 719, 695, $647,638,555,544,535,480,443,413 \mathrm{~cm}^{-1}$. HRMS (APCI): $\mathrm{m} / z$ calcd for $\mathrm{C}_{45} \mathrm{H}_{32} \mathrm{~N}[\mathrm{M}+\mathrm{H}]^{+}$586.2529, found 586.2530.

2,3,4,5,6-Pentaphenyl-1-(quinolin-4-yl)benzene (10c). 2,3,4,5,6-Pentaphenyl-1-(quinoline-4-yl)benzene 10c was prepared by Procedure 2 using 4-(phenylethynyl)quinoline 8c (0.302 g, $1.319 \mathrm{mmol})$ and tetraphenylcyclopentadienone 9 ( $0.430 \mathrm{~g}, 1.121 \mathrm{mmol}, 0.85$ equiv.) in benzophenone (10 g) in $50 \mathrm{~mL}$ round-bottomed flask in $0.5 \mathrm{~h}$ (total conversion of dienone). Yield $0.529 \mathrm{~g}$, 81\%, a white solid, m.p. $323{ }^{\circ} \mathrm{C} .{ }^{1} \mathrm{H}$ NMR (600 MHz, $\mathrm{CDCl}_{3}$ ): $\delta=8.49(\mathrm{~d}, J=4.4 \mathrm{~Hz}, 1 \mathrm{H}, 2-\mathrm{H}), 7.86$ (dd, $J=0.5,7.9 \mathrm{~Hz}, 1 \mathrm{H}, 5-\mathrm{H}), 7.80$ (d, $J=8.3,1 \mathrm{H}, 8-\mathrm{H}), 7.50$ (ddd, $J=1.5,6.9,8.4 \mathrm{~Hz}, 1 \mathrm{H}, 7-\mathrm{H}), 7.39$ (ddd, $J=1.3,6.9,8.3$ $\mathrm{Hz}, 1 \mathrm{H}, 6-\mathrm{H}), 7.05$ (d, $J=4.4 \mathrm{~Hz}, 1 \mathrm{H}, 3-\mathrm{H}), 6.93-6.82(\mathrm{~m}, 17 \mathrm{H}$, $\mathrm{Ph})$, 6.77-6.75 (m, 2H, Ph), 6.68-6.64 (m, 4H, Ph), 6.55-6.52 $(\mathrm{m}, 2 \mathrm{H}, \mathrm{Ph}) \mathrm{ppm} .{ }^{13} \mathrm{C} \mathrm{NMR}\left(150 \mathrm{MHz}, \mathrm{CDCl}_{3}\right): \delta=148.8(+$, C2), 147.9 (o, C4), 147.7 (o, C8a), 141.5 (o), 140.74 (o), 140.70 (o), 140.4 (o), 140.2 (o), 139.7 (o), 136.0 (o, C $\alpha), 131.53$ $(+), 131.46(+), 131.44(+), 130.9(+), 130.2(+), 129.3(+$, C8), 128.8 (+, C7), 128.0 (o, C4a), 127.2 (+, C5), $126.84(+), 126.81$ $(+), 126.7(+), 126.6(+), 125.8(+), 125.7(+, \mathrm{C} 6), 125.5(+), 124.4$ $(+$, C3) ppm. IR (ATR): 3052, 3025, 1598, 1496, 1440, 1385, 1291, 1071, 1028, 905, 852, 817, 759, 736, 718, 695, 616, 586, $562,551,542,463,449,425 \mathrm{~cm}^{-1}$. HRMS (ESI): $\mathrm{m} / \mathrm{z}$ calcd for $\mathrm{C}_{45} \mathrm{H}_{32} \mathrm{~N}[\mathrm{M}+\mathrm{H}]^{+}$586.2530, found 586.2522.

3-((4-Ethynylphenyl)ethynyl)quinoline (14). According to Procedure 1, a solution of $2.080 \mathrm{~g}(10.00 \mathrm{mmol})$ of 3-bromoquinoline $6 \mathbf{b}, 0.070 \mathrm{~g}(0.10 \mathrm{mmol})$ of $\mathrm{Pd}\left(\mathrm{PPh}_{3}\right)_{2} \mathrm{Cl}_{2}, 0.038 \mathrm{~g}$ $(0.20 \mathrm{mmol})$ of $\mathrm{CuI}$ and $1.197 \mathrm{~g}(9.50 \mathrm{mmol})$ of 1,4-diethynylbenzene 13 in $50 \mathrm{~mL}$ of anhydrous $\mathrm{NEt}_{3}$ was heated $(3.5 \mathrm{~h})$ under reflux temperature. Finally, a purification by column chromatography (petroleum ether: ethyl acetate $=3: 1$ ) gave 3-((4-ethynylphenyl)ethynyl)quinoline 14. Yield $0.889 \mathrm{~g}, 37 \%$, a brown solid, m.p. $113{ }^{\circ} \mathrm{C} .{ }^{1} \mathrm{H}$ NMR (600 MHz, $\mathrm{CDCl}_{3}$ ): $\delta=8.99$ $(\mathrm{d}, J=2.1 \mathrm{~Hz}, 1 \mathrm{H}, 2-\mathrm{H}), 8.30(\mathrm{~d}, J=2.1 \mathrm{~Hz}, 1 \mathrm{H}, 4-\mathrm{H}), 8.10(\mathrm{~d}, J=$ $8.5 \mathrm{~Hz}, 1 \mathrm{H}, 8-\mathrm{H}), 7.70(\mathrm{~d}, J=8.1 \mathrm{~Hz}, 1 \mathrm{H}, 5-\mathrm{H}), 7.73$ (ddd, $J=$ 1.5, 7.0, $8.5 \mathrm{~Hz}, 1 \mathrm{H}, 7-\mathrm{H}$ ), 7.57 (ddd, $J=1.5,7.0,8.1 \mathrm{~Hz}, 1 \mathrm{H}$, 6-H), 7.55-7.53 (m, 2H, 2'-H, 6'-H), 7.51-7.49 (m, 2H, 3'-H, 5'$\mathrm{H}), 3.20(\mathrm{~s}, 1 \mathrm{H}, \mathrm{CCH}) \mathrm{ppm} .{ }^{13} \mathrm{C} \mathrm{NMR}\left(150 \mathrm{MHz}, \mathrm{CDCl}_{3}\right): \delta=$ 152.1 (+, C2), 147.1 (o, C8a), 138.5 (+, C4), 132.3 (+, C3', C5'), 131.7 (+, C2', C6'), 130.4 (+, C7), 129.6 (+, C8), 127.8 (+, C5), 127.5 (+, C6), 127.4 (o, C4a), 123.2 (o, C1'), 122.6 (o, C4'), 117.2

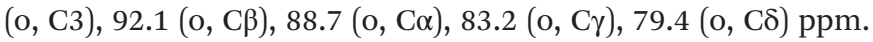
IR (ATR): 3265, 3060, 3034, 2101, 1969, 1710, 1699, 1602, 1566, 1487, 1404, 1351, 1266, 1145, 1105, 1010, 981, 958, 906, 861, 838, 782, 752, 691, 653, 622, 548, 471, $419 \mathrm{~cm}^{-1}$. HRMS (ESI): $\mathrm{m} / \mathrm{z}$ calcd for $\mathrm{C}_{19} \mathrm{H}_{12} \mathrm{~N}[\mathrm{M}+\mathrm{H}]^{+} 254.0964$, found $254.0972 ; \mathrm{m} / \mathrm{z}$ calcd for $\mathrm{C}_{19} \mathrm{H}_{11} \mathrm{NNa}[\mathrm{M}+\mathrm{Na}]^{+} 276.0789$, found 276.0785 .

1-(Quinolin-3-yl)-2,3,4,5,-tetraphenyl-6-(4-(1,2,3,4-tetraphenyl) phenyl)-benzene (15). 1-(Quinolin-3-yl)-2,3,4,5,-tetraphenyl-6(4-(1,2,3,4-tetraphenyl)phenyl)benzene $\mathbf{1 5}$ was prepared by Procedure 2 using 3-((4-ethynylphenyl)ethynyl)quinoline 14 $(0.290 \mathrm{~g}, 1.146 \mathrm{mmol})$ and tetraphenylcyclopentadienone 9 (1.320 g, $3.438 \mathrm{mmol}, 3.0$ equiv.) in benzophenone (10 g) in $50 \mathrm{~mL}$ round-bottomed flask in $0.5 \mathrm{~h}$. Yield $0.885 \mathrm{~g}, 77 \%$, a 
brown solid, m.p. $368{ }^{\circ} \mathrm{C}$ (decomp.). ${ }^{1} \mathrm{H}$ NMR $(600 \mathrm{MHz}$, $\left.\mathrm{CDCl}_{3}\right): \delta=8.42(\mathrm{~d}, J=2.0 \mathrm{~Hz}, 1 \mathrm{H}), 7.90(\mathrm{~d}, J=8.3 \mathrm{~Hz}, 1 \mathrm{H})$, 7.57 (ddd, $J=1.4,6.8,8.3 \mathrm{~Hz}, 1 \mathrm{H}), 7.55$ (d, $J=2.0 \mathrm{~Hz}, 1 \mathrm{H}$ ), 7.46-7.45 (m, 1H), 7.41-7.38 (m, 1H), 7.23 (s, 1H), 7.15-1.13 (m, 2H), 7.07-7.06 (m, 2H), 6.94-6.55 (m, 40H) ppm. ${ }^{13} \mathrm{C}$ NMR (150 MHz, $\mathrm{CDCl}_{3}$ ): $\delta=152.9(+), 145.7$ (o), 141.8 (o), 141.7 (o), 141.3 (o), 141.1 (o), 140.86 (o), 140.82 (o), 140.80 (o), 140.73 (o), 140.48 (o), 140.43 (o), 140.34 (o), 140.27 (o), 140.21 (o), 140.07 (o), 139.95 (o), 139.7 (o), 139.3 (o), 139.1 (o), 138.8 (o), 137.9 (o), $137.8(+), 136.5$ (o), 134.2 (o), $132.5(+), 131.6(+)$, $131.5(+), 131.39(+), 131.35(+), 131.0(+), 130.9(+), 130.2(+)$, $130.0(+), 129.1(+), 128.89(+), 128.87(+), 128.77(+), 128.4(+)$, $127.69(+), 127.62(+), 127.3(+), 127.1(+), 127.0(+), 126.96(\mathrm{o})$, $126.82(+), 126.76(+), 126.66(+), 126.31(+), 126.2(+), 125.8(+)$, $125.64(+), 125.55(+), 125.50(+), 125.46(+), 125.36(+)$ ppm. IR (ATR): 3024, 1599, 1490, 1441, 1398, 1275, 1071, 1021, 907, 851, 763, 696, 638, 615, 566, 554, 480, $425 \mathrm{~cm}^{-1}$. HRMS (ESI): $m / z$ calcd for $\mathrm{C}_{75} \mathrm{H}_{51} \mathrm{NNa}[\mathrm{M}+\mathrm{Na}]^{+}$988.3914, found 988.3901.

3,3'-Ethyne-1,2-diyldiquinoline (18a). According to Procedure 1, a solution of $0.208 \mathrm{~g}(1.00 \mathrm{mmol})$ of 3-bromoquinoline $6 \mathbf{b}, 0.007 \mathrm{~g}(0.01 \mathrm{mmol})$ of $\mathrm{Pd}\left(\mathrm{PPh}_{3}\right)_{2} \mathrm{Cl}_{2}, 0.0038 \mathrm{~g}$ $(0.02 \mathrm{mmol}) \mathrm{CuI}$ and $0.2184 \mathrm{~g}(1.05 \mathrm{mmol})$ of 3-ethynylquinoline 17 in $10 \mathrm{~mL}$ of anhydrous $\mathrm{NEt}_{3}$ was heated $(2 \mathrm{~h})$ under reflux temperature. Finally, a purification by column chromatography (petroleum ether: ethyl acetate $=3: 1$ ) gave 3,3'ethyne-1,2-diyldiquinoline 18a. Yield $0.196 \mathrm{~g}, 70 \%$, a brownish solid, m.p. $173{ }^{\circ} \mathrm{C} .{ }^{1} \mathrm{H}$ NMR (600 MHz, $\left.\mathrm{CDCl}_{3}\right): \delta=9.05$ (d, $J=$ $\left.2 \mathrm{~Hz}, 2 \mathrm{H}, 2-\mathrm{H}, 2^{\prime}-\mathrm{H}\right), 8.36$ (d, $\left.J=2 \mathrm{~Hz}, 2 \mathrm{H}, 4-\mathrm{H}, 4^{\prime}-\mathrm{H}\right), 8.12$ (d, $\left.J=8.1 \mathrm{~Hz}, 2 \mathrm{H}, 8-\mathrm{H}, 8^{\prime}-\mathrm{H}\right), 7.82$ (d, $\left.J=8.3 \mathrm{~Hz}, 2 \mathrm{H}, 5-\mathrm{H}, 5^{\prime}-\mathrm{H}\right)$, $7.74\left(\mathrm{ddd}, J=1.4,6.9,8.4 \mathrm{~Hz}, 2 \mathrm{H}, 7-\mathrm{H}, 7^{\prime}-\mathrm{H}\right), 7.58$ (ddd, $J=1.1$, 6.9, 8.0 Hz, 2H, 6-H, 6'-H) ppm. ${ }^{13} \mathrm{C} \mathrm{NMR} \mathrm{(150} \mathrm{MHz,} \mathrm{CDCl}_{3}$ ): $\delta=152.0\left(+, \mathrm{C} 2, \mathrm{C} 2{ }^{\prime}\right), 147.2\left(\mathrm{o}, \mathrm{C} 8 \mathrm{a}, \mathrm{C} 8 \mathrm{a}^{\prime}\right), 138.8\left(+, \mathrm{C} 4, \mathrm{C} 4{ }^{\prime}\right)$, $130.5\left(+, \mathrm{C} 7, \mathrm{C} 7^{\prime}\right), 129.6\left(+, \mathrm{C} 8, \mathrm{C} 8^{\prime}\right), 127.8\left(+, \mathrm{C} 5, \mathrm{C} 5^{\prime}\right), 127.6(+$, C6, C6'), 127.3 (o, C4a, C4a'), 116.9 (o, C3, C3'), 90.0 (o, C $\alpha$ ) ppm. IR (ATR): 3061, 2169, 1839, 1616, 1565, 1487, 1468, 1413, 1357, 1291, 1226, 1192, 1122, 991, 955, 906, 878, 869, 786, 770, 761, 748, 737, 640, 614, 594, 553, 527, 491, 479, 472, 461, 445, $415 \mathrm{~cm}^{-1}$. MS (ESI): $m / z=281.1[\mathrm{M}+\mathrm{H}]^{+}$. HRMS (ESI): $\mathrm{m} / z$ calcd for $\mathrm{C}_{20} \mathrm{H}_{13} \mathrm{~N}_{2}[\mathrm{M}+\mathrm{H}]^{+}$281.1074, found 281.1072.

3-(Quinolin-4-ylethynyl)quinoline (18b). According to Procedure 1, a solution of $0.208 \mathrm{~g}(1.00 \mathrm{mmol})$ of 4-bromoquinoline 6c, $0.070 \mathrm{~g}(0.10 \mathrm{mmol})$ of $\mathrm{Pd}\left(\mathrm{PPh}_{3}\right)_{2} \mathrm{Cl}_{2}, 0.038 \mathrm{~g}$ $(0.20 \mathrm{mmol}) \mathrm{CuI}$ and $0.218 \mathrm{~g}(1.05 \mathrm{mmol})$ of 3-ethynylquinoline $17 \mathrm{in} 10 \mathrm{~mL}$ of anhydrous $\mathrm{NEt}_{3}$ was heated $(3.5 \mathrm{~h})$ under reflux temperature. Finally, a purification by column chromatography (petroleum ether : ethyl acetate $=3: 1$ ) gave 3-(quinolin-4-ylethynyl)quinoline 18b. Yield $0.160 \mathrm{~g}, 57 \%$, a yellow solid, m.p. $134{ }^{\circ} \mathrm{C} .{ }^{1} \mathrm{H}$ NMR (600 MHz, $\left.\mathrm{CDCl}_{3}\right): \delta=9.11$ (d, $J=$ $2.1 \mathrm{~Hz}, 1 \mathrm{H}, 2-\mathrm{H}), 8.94$ (d, $\left.J=4.5 \mathrm{~Hz}, 1 \mathrm{H}, 2^{\prime}-\mathrm{H}\right), 8.45$ (d, $J=$ $2.0 \mathrm{~Hz}, 1 \mathrm{H}, 4-\mathrm{H}), 8.40\left(\mathrm{dd}, J=0.9,8.3 \mathrm{~Hz}, 1 \mathrm{H}, 5^{\prime}-\mathrm{H}\right), 8.16(\mathrm{~d}, J=$ $\left.7.8,1 \mathrm{H}, 8^{\prime}-\mathrm{H}\right), 8.14$ (d, $\left.J=7.8 \mathrm{~Hz}, 1 \mathrm{H}, 8-\mathrm{H}\right), 7.85$ (d, $J=7.9 \mathrm{~Hz}$, $1 \mathrm{H}, 4-\mathrm{H}$ ), $7.80-7.76$ (m, 2H, 7-H, 7'-H), 7.68 (ddd, $J=1.2,6.9$, $\left.8.2 \mathrm{~Hz}, 1 \mathrm{H}, 66^{\prime}-\mathrm{H}\right), 7.63$ (d, $\left.J=4.3 \mathrm{~Hz}, 1 \mathrm{H}, 3^{\prime}-\mathrm{H}\right), 7.61$ (ddd, $J=$ $1.2,7.1,8.2 \mathrm{~Hz}, 1 \mathrm{H}, 6-\mathrm{H}) \mathrm{ppm} .{ }^{13} \mathrm{C} \mathrm{NMR}\left(150 \mathrm{MHz}, \mathrm{CDCl}_{3}\right): \delta=$ 151.9 (+, C2), 149.9 (+, C2'), 148.3 (o, C8a'), 147.5 (o, C8a), 139.2 (+, C4), 130.9 (+, C7), 130.2 (+, C7'), 130.2 (+, C8'), 129.7
(+, C8), 129.1 (o, C4'), 127.9 (+, C5), 127.7 (+, C6), 127.6 (o, C4a'), $127.6\left(+, \mathrm{C}^{\prime}\right), 127.3$ (o, C4a), $126.0\left(+, \mathrm{C}^{\prime}\right), 123.9(+$, C3') 116.5 (o, C3), 95.7 (o, C $\alpha$ ), 88.3 (o, C $\beta$ ) ppm. IR (ATR): 3033, 2209, 2203, 1615, 1572, 1484, 1419, 1282, 1220, 1192, 1123, 1011, 976, 905, 845, 787, 758, 651, 609, 542, 514, 491, 476, $445 \mathrm{~cm}^{-1}$. MS (ESI): $m / z=281.1[\mathrm{M}+\mathrm{H}]^{+}$. HRMS (ESI): $\mathrm{m} / z$ calcd for $\mathrm{C}_{20} \mathrm{H}_{13} \mathrm{~N}_{2}[\mathrm{M}+\mathrm{H}]^{+}$281.1074, found 281.1062.

1,2-Di(quinolin-3-yl)-3,4,5,6-tetraphenylbenzene

(19a). According to Procedure 2, benzophenone (5 g) was melted in a $50 \mathrm{~mL}$ round-bottomed flask fitted with an air condenser. 3,3'Ethyne-1,2-diyldiquinoline 18a $(0.280 \mathrm{~g}, 1.00 \mathrm{mmol})$ and tetraphenyl-cyclopentadienone 9 (0.576 g, $1.50 \mathrm{mmol})$ were added to the flask, which was heated for $1 \mathrm{~h}$ using an open flame. The solution was cooled to $\mathrm{rt}$ and toluene $(3 \mathrm{~mL})$ was added to prevent the solidification of the benzophenone. After cooling, $n$-hexane $(200 \mathrm{~mL})$ was added, resulting in the precipitation of 19a as a white powder, which was washed with $n$-hexane and toluene, and collected by vacuum filtration. Yield $0.570 \mathrm{~g}$, $90 \%$, a white solid, m.p. $344{ }^{\circ} \mathrm{C} .{ }^{1} \mathrm{H}$ NMR $(600 \mathrm{MHz}$, DMSO- $\left.d_{6}\right)$, two sets of isochronous rotameric forms $(1: 0.8)$ : $\delta=8.52\left(\mathrm{~d}, J=2.1 \mathrm{~Hz}, 3.6 \mathrm{H}, 2-\mathrm{H}, 2^{\prime}-\mathrm{H}\right), 7.97(\mathrm{~d}, J=1.7 \mathrm{~Hz}, 2 \mathrm{H}$, $4-\mathrm{H}), 7.93$ (d, $\left.J=1.7 \mathrm{~Hz}, 1.6 \mathrm{H}, 4^{\prime}-\mathrm{H}\right), 7.64-7.61(\mathrm{~m}, 3.6 \mathrm{H}, 8-\mathrm{H}$, $\left.8^{\prime}-\mathrm{H}\right), 7.59$ (d, $\left.J=7.9 \mathrm{~Hz}, 2 \mathrm{H}, 5-\mathrm{H}\right), 7.52(\mathrm{~d}, J=8.3 \mathrm{~Hz}, 1.6 \mathrm{H}$, 5'-H), 7.50-7.47 (m, 3.6H, 7-H, 7'-H), 7.38-7.36 (m, 2H, 6-H), 7.35-7.32 (m, 1.6H, 6'-H), 7.01-6.74 (m, 36H, Ph) ppm. ${ }^{13} \mathrm{C}$ NMR (150 MHz, DMSO- $\left.d_{6}\right): \delta=152.0(+, \mathrm{C} 2), 151.8\left(+, \mathrm{C}^{\prime}\right)$, 144.99 (o, C8a'), 144.98 (o, C8), 141.06 (o), 141.04 (o), 140.9 (o), 139.5 (o), 139.29 (o), 139.25 (o), 137.4 (+, C4'), 137.0 (+, C4), 136.95 (o, C $\alpha$ ), 136.93 (o, C $\beta$ ), 132.97 (o), 132.94 (o), 130.93 (+), $130.89(+), 130.77(+), 130.72(+), 130.70(+), 129.66(+)$, $129.51(+), 129.18(+, \mathrm{C} 7$ or C7'), 129.16 (+, C7 or C7'), 128.34 (+, C8 or C8'), 128.31 (+, C8 or C8'), 127.8 (+, C5), $127.6\left(+, \mathrm{C}^{\prime}\right)$, $127.0(+), 126.8(+), 126.7(+), 126.5\left(+, \mathrm{C} 6, \mathrm{C6}^{\prime}\right), 126.3$ (o, C4a or $\left.\mathrm{C}_{4} \mathrm{a}^{\prime}\right), 126.20\left(\mathrm{o}, \mathrm{C} 4 \mathrm{a}\right.$ or $\left.\mathrm{C} 4 \mathrm{a}^{\prime}\right), 125.9(+), 125.7(+)$ ppm. IR (ATR): 3055, 3025, 1600, 1552, 1490, 1401, 1314, 1241, 1127, 1071, 966, 907, 812, 784, 747, 696, 632, 536, $476 \mathrm{~cm}^{-1}$. HRMS (ESI): $m / z$ calcd for $\mathrm{C}_{48} \mathrm{H}_{33} \mathrm{~N}_{2}[\mathrm{M}+\mathrm{H}]^{+} 637.2639$, found 637.2637 .

1-(Quinolin-3-yl)-2-(quinolin-4-yl)-3,4,5,6-tetraphenylbenzene (19b). 1-(Quinoline-3-yl)-2-(quinoline-4-yl)-3,4,5,6-tetraphenylbenzene 19b was prepared by Procedure 2 using 4-(quinolin-3ylethynyl)quinoline $18 \mathrm{~b}(0.264 \mathrm{~g}, 0.943 \mathrm{mmol})$ and tetraphenylcyclopentadienone $9(0.543 \mathrm{~g}, 1.414 \mathrm{mmol})$ in benzophenone $(5 \mathrm{~g})$ in $10 \mathrm{~mL}$ round-bottomed flask in $0.5 \mathrm{~h}$. The solution was cooled to $\mathrm{rt}$ and toluene $(4 \mathrm{~mL})$ was added to prevent the solidification of the benzophenone. Then solvents were evaporated with silica gel and obtained solid was purified by column chromatography with EE: PE $(1: 1)$ as eluents to give 19b. Yield $0.469 \mathrm{~g}, 78 \%$, a white solid, m.p. $300{ }^{\circ} \mathrm{C}$ (decomp.). ${ }^{1} \mathrm{H}$ NMR (600 MHz, DMSO- $\left.d_{6}\right): \delta=8.58(\mathrm{~d}, J=2.2 \mathrm{~Hz}, 1 \mathrm{H})$, 8.46-8.44 (m, 2H), 8.25 (d, $J=2.2 \mathrm{~Hz}, 1 \mathrm{H}), 7.97-7.96(\mathrm{~m}, 1 \mathrm{H})$, 7.91-7.87 (m, 2H), 7.61-7.49 (m, 10H), 7.47 (ddd, $J=1.6,6.8$, $8.4 \mathrm{~Hz}, 2 \mathrm{H}), 7.45$ (ddd, $J=1.5,6.6,8.2 \mathrm{~Hz}, 1 \mathrm{H}), 7.41-7.39$ (m, $2 \mathrm{H}), 7.36$ (ddd, $J=1.2,6.8,8.1 \mathrm{~Hz}, 1 \mathrm{H}), 7.26-7.24(\mathrm{~m}, 1 \mathrm{H})$, 7.21-7.19 (m, 1H), 7.13-7.00 (m, 8H), 6.96-6.81 (m, 22H), 6.79-6.72 (m, 3H), 6.71-6.67 (m, 4H), $6.55(\mathrm{t}, J=7.6 \mathrm{~Hz}, 2 \mathrm{H})$ 
ppm. ${ }^{13} \mathrm{C}$ NMR (150 MHz, DMSO- $\left.d_{6}\right): \delta=151.50(+), 150.81(+)$, 148.76 (+), 148.58 (+), 146.85 (o), 146.78 (o), 145.98 (o), 145.89 (o), 145.00 (o), 144.90 (o), 141.21 (o), 141.18 (o), 141.07 (o), 141.01 (o), 140.86 (o), 140.70 (o), 140.29 (o), 140.20 (o), 139.52 (o), 139.45 (o), 139.17 (o), 139.15 (o), 139.05 (o), 138.94 (o), 136.72 (+), 136.35 (o), 136.29 (o), 135.89 (o), 135.82 (o), $135.61(+), 132.66$ (o), 132.55 (o), 131.11 (+), $130.98(+)$, $130.94(+), 130.90(+), 130.81(+), 130.76(+), 130.71(+), 130.63$ $(+), 130.60(+), 130.58(+), 130.53(+), 130.42(+), 129.36(+)$, $129.07(+), 129.01(+), 128.95(+), 128.75(+), 128.68(+), 128.21$ $(+), 127.63(+), 127.31(+), 126.93(+), 126.89(+), 126.81$ $(+), 126.76(+), 126.72(+), 126.67(+), 126.63(+), 126.49(+)$, $126.45(+), 126.40(+), 126.33(+), 126.24(+), 125.89(+), 125.83$ $(+), 125.73(+), 125.65(+), 124.37(+), 123.82(+)$ ppm. IR (ATR): 3055, 3027, 1699, 1600, 1585, 1567, 1505, 1490, 1463, 1442, 1384, 1359, 1126, 1072, 1027, 965, 908, 854, 814, 761, 696, 617, 533, $480 \mathrm{~cm}^{-1}$. HRMS (ESI): $m / z$ calcd for $\mathrm{C}_{48} \mathrm{H}_{33} \mathrm{~N}_{2}[\mathrm{M}+\mathrm{H}]^{+}$ 637.2639 , found 637.2629 .

\section{General procedure for the synthesis of substituted benzenes (Procedure 3)}

2-(Phenylethynyl)quinoline $\mathbf{8 a}(0.21 \mathrm{~g}, 0.92 \mathrm{mmol})$ was dissolved in anhydrous dioxane $(4 \mathrm{~mL})$ in an oven dried Schlenk flask under a nitrogen atmosphere and the flask was evacuated and filled with nitrogen repeatedly $(3 \times)$. Then, $\mathrm{Co}_{2}(\mathrm{CO})_{8}$ $(0.015 \mathrm{~g})$ was added to the flask under a nitrogen atmosphere and the flask was evacuated and filled with nitrogen again $(3 \times)$. The resulting mixture was refluxed for $14 \mathrm{~h}$ and dioxane was evaporated. The resulting residue was dissolved in dichloromethane and filtered through a short pad of silica gel. Evaporation of the solvent afforded a dark colored solid which was purified by column chromatography with PE-EE as an eluent.

1,3,5-Triphenyl-2,4,6-tri(quinolin-2-yl)benzene (20a). Yield $0.021 \mathrm{~g}, 10 \%$, a white solid, m.p. $337{ }^{\circ} \mathrm{C} .{ }^{1} \mathrm{H}$ NMR $(600 \mathrm{MHz}$, DMSO- $\left.d_{6}\right): \delta=7.83\left(\mathrm{~d}, J=8.5 \mathrm{~Hz}, 3 \mathrm{H}, 4^{\prime}-\mathrm{H}\right), 7.72(\mathrm{~d}, J=8.5 \mathrm{~Hz}$, $\left.3 \mathrm{H}, 8^{\prime}-\mathrm{H}\right), 7.67$ (d, $\left.J=7.9 \mathrm{~Hz}, 3 \mathrm{H}, 5^{\prime}-\mathrm{H}\right), 7.57$ (t, $J=7.3 \mathrm{~Hz}, 3 \mathrm{H}$, $\left.7^{\prime}-\mathrm{H}\right), 7.41$ (t, $\left.J=7.3 \mathrm{~Hz}, 3 \mathrm{H}, 6^{\prime}-\mathrm{H}\right), 7.22$ (d, $\left.J=8.5 \mathrm{~Hz}, 3 \mathrm{H}, 3^{\prime}-\mathrm{H}\right)$, 7.05 (br s, 6H, 2"-H, 6"-H), 6.70 (br s, 6H, 3"--H, 5"-H), 6.63 (t, $\left.J=7.3 \mathrm{~Hz}, 3 \mathrm{H}, 4^{\prime \prime}-\mathrm{H}\right)$ ppm. ${ }^{13} \mathrm{C}$ NMR (150 MHz, DMSO- $\left.d_{6}\right): \delta=$ 158.9 (o, C2'), 146.4 (o, 8a'), 139.9 (o, C2, C4; C6), 139.8 (o, C1, C3, C5), 138.7 (o, C1"), 134.1 (+, C4'), 130.6 (+, C2", C6"), 129.0 (+, C7'), 128.6 (+, C8'), 127.4 (+, C5'), 126.3 (+, C3", C5"), 126.1 (+, C6'), 125.8 (+, C4"), 125.4 (o, C4a'), 124.2 (+, C3') ppm. IR (ATR): 3058, 3038, 3007, 1617, 1595, 1557, 1501, 1443, 1424, 1332, 1305, 1253, 1223, 1156, 1139, 1113, 1073, 1031, 959, 906, 838, 753, 698, 619, 587, 515, 476, 431, 423, $412 \mathrm{~cm}^{-1}$. MS (ESI): $m / z=688.5[\mathrm{M}+\mathrm{H}]^{+}$. HRMS (ESI): $m / z$ calcd for $\mathrm{C}_{51} \mathrm{H}_{34} \mathrm{~N}_{3}$ $[\mathrm{M}+\mathrm{H}]^{+} 688.2745$, found 688.2743.

1,2,4-Triphenyl-3,5,6-tri(quinolin-2-yl)benzene (20b). Yield $0.063 \mathrm{~g}, 30 \%$, a white solid, m.p. $318{ }^{\circ} \mathrm{C} .{ }^{1} \mathrm{H}$ NMR $(600 \mathrm{MHz}$, DMSO- $\left.d_{6}\right): \delta=7.85(\mathrm{~d}, J=7.5 \mathrm{~Hz}, 1 \mathrm{H}), 7.76-7.68(\mathrm{~m}, 4 \mathrm{H})$, 7.57-7.53 (m, 5H), 7.45-7.42 (m, 3H), 7.32-7.29 (m, 2H), 7.22-7.20 (m, 3H), 7.04-6.99 (m, 6H), 6.83-6.67 (m, 9H) ppm. ${ }^{13} \mathrm{C}$ NMR (150 MHz, DMSO- $d_{6}$ ): $\delta=159.1$ (o), 159.0 (o), 158.8 (o), 146.4 (o), 146.3 (o), 146.2 (o), 140.5 (o), 140.4 (o), 140.2 (o),
139.8 (o), 139.6 (o), 139.3 (o), 139.0 (o), 138.9 (o), 138.6 (o), $134.1(+), 133.6(+), 130.8(+), 130.3(\mathrm{br},+), 129.0(+), 128.8(+)$, $128.6(+), 128.3(+), 127.4(+), 127.3(+), 126.6(+), 126.5(+)$, $126.1(+), 125.92(+), 125.85(+), 125.81(+), 125.7(+), 125.3(\mathrm{o})$, 125.2 (o), $124.3(+), 124.2(+)$ ppm. IR (ATR): 3056, 3023, 1737, 1597, 1559, 1501, 1441, 1424, 1294, 1160, 1142, 1294, 1160, 1142, 1111, 1072, 1029, 942, 849, 835, 818, 751, 722, 699, 606, 531, 514, 479, 423, $417 \mathrm{~cm}^{-1}$. MS (ESI): $m / z=688.3[\mathrm{M}+\mathrm{H}]^{+}$. HRMS (ESI): $m / z$ calcd for $\mathrm{C}_{51} \mathrm{H}_{34} \mathrm{~N}_{3}[\mathrm{M}+\mathrm{H}]^{+} 688.2745$, found 688.2739 .

Hexakis(quinolin-3-yl)benzene (21). Hexakis(quinoline-3-yl) benzene 11 was prepared by an identical procedure (Procedure 3) using bis (quinoline-3-yl)acetylene 18a (0.295 g, $0.35 \mathrm{mmol})$ as above. A dark colored solid was purified by column chromatography with $\mathrm{EE}$, then $\mathrm{MeOH}-\mathrm{CHCl}_{3}(1: 3)$ as eluents. Yield $0.295 \mathrm{~g}, 85 \%$, a white solid, m.p. $>370^{\circ} \mathrm{C}$. The compound is insoluble in all NMR solvents which we tested. IR (ATR): 3025, 1619, 1602, 1567, 1489, 1355, 1317, 1260, 1195, 1126, 1110, 1043, 1017, 974, 953, 905, 858, 785, 744, 696, 605, 527, 474, $439 \mathrm{~cm}^{-1}$. HRMS (ESI): $\mathrm{m} / z$ calcd for $\mathrm{C}_{60} \mathrm{H}_{36} \mathrm{~N}_{6} \mathrm{Na}[\mathrm{M}+\mathrm{Na}]^{+}$ 863.2899, found 863.2896.

\section{General procedure for the preparation of the salts (Procedure 4)}

Samples of $0.50 \mathrm{mmol}$ of the corresponding quinolines were dissolved in toluene containing 1 drop of nitrobenzene. Then $0.75 \mathrm{mmol}$ of dimethyl sulfate was added with stirring. Thereafter the resulting mixture was stirred under reflux temperature. After completion of the reaction (controlled by TLC), the solution was cooled, the crude product was filtered off, washed with ethyl acetate $(3 \times 10 \mathrm{~mL})$, and dried to afford the product.

2,3,4,5,6-Pentaphenyl-1-(1-methylquinolinium-2-yl)benzene methylsulfate (22a). According to Procedure 4, a solution of $0.100 \mathrm{~g}$ (0.171 $\mathrm{mmol})$ of 2,3,4,5,6-pentaphenyl-1-(quinoline-2yl)benzene 10a, 1 drop of nitrobenzene and $0.06 \mathrm{~mL}$ $(0.63 \mathrm{mmol})$ of dimethyl sulfate in $5 \mathrm{~mL}$ of anhydrous toluene was heated for $2 \mathrm{~h}$ under reflux temperature to give 2,3,4, 5,6-pentaphenyl-1-(1-methylquinolinium-2-yl)benzene methylsulfate 22a. Yield $0.115 \mathrm{~g}, 95 \%$, a white solid, m.p. $190{ }^{\circ} \mathrm{C} .{ }^{1} \mathrm{H}$ NMR (600 MHz, DMSO- $\left.d_{6}\right): \delta=8.88\left(\mathrm{~d}, J=8.5 \mathrm{~Hz}, 1 \mathrm{H}, 4^{\mathrm{i}}-\mathrm{H}\right)$, $8.31\left(\mathrm{~d}, J=9.0 \mathrm{~Hz}, 1 \mathrm{H}, 8^{\mathrm{i}}-\mathrm{H}\right), 8.24-8.23\left(\mathrm{~m}, 2 \mathrm{H}, 3^{\mathrm{i}}-\mathrm{H}, 5^{\mathrm{i}}-\mathrm{H}\right), 8.14$ $\left(\mathrm{t}, J=8.0 \mathrm{~Hz}, 7^{\mathrm{i}}-\mathrm{H}\right), 7.93\left(\mathrm{t}, J=7.4 \mathrm{~Hz}, 6^{\mathrm{i}}-\mathrm{H}\right), 7.10-6.84(\mathrm{~m}, 25 \mathrm{H}$, $\mathrm{Ph}), 4.41$ (s, $3 \mathrm{H}, \mathrm{N}^{\mathrm{i}} \mathrm{CH}_{3}$ ), 3.38 (s, 3H, $\mathrm{CH}_{3} \mathrm{SO}_{4}$ ) ppm. ${ }^{13} \mathrm{C} \mathrm{NMR}$ (150 MHz, DMSO- $d_{6}$ ): $\delta=159.1\left(\mathrm{o}, \mathrm{C} 2^{\mathrm{i}}\right), 144.2\left(+, \mathrm{C} 4^{\mathrm{i}}\right), 143.9$ (o, C1), 141.2 (o), 139.1 (o), 138.9 (o), 138.3 (o), 137.5 (o, C8a ${ }^{\mathrm{i}}$ ), $137.4(\mathrm{o}), 136.0\left(+, \mathrm{C7}^{\mathrm{i}}\right), 131.7(+), 130.9(+), 130.6(+), 130.4(+)$, $130.4\left(+, \mathrm{C}^{\mathrm{i}}\right), 130.3\left(+, \mathrm{C}^{\mathrm{i}}\right), 130.26(+), 130.0(+), 129.3(+)$, $126.7(+), 127.43\left(\mathrm{o}, \mathrm{C} 4 \mathrm{a}^{\mathrm{i}}\right), 127.39\left(+, \mathrm{C} 3^{\mathrm{i}}\right), 127.2(+), 126.95(+)$, $126.91(+), 126.86(+), 126.2(+), 126.0(+), 119.1\left(+, \mathrm{C}^{\mathrm{i}}\right), 52.8$ $\left(+, \mathrm{CH}_{3} \mathrm{SO}_{4}\right), 42.5\left(+, \mathrm{N}^{\mathrm{i}} \mathrm{CH}_{3}\right)$ ppm. IR (ATR): 3056, 3023, 1619, $1600,1577,1519,1497,1442,1409,1347,1252,1224,1176$, 1152, 1073, 1059, 1013, 918, 849, 817, 770, 756, 723, 698, 644, 608, 576, 554, 532, $428 \mathrm{~cm}^{-1}$. HRMS (ESI): $\mathrm{m} / \mathrm{z}$ calcd for $\mathrm{C}_{46} \mathrm{H}_{34} \mathrm{~N}[\mathrm{M}]^{+}$600.2686, found 600.2692.

2,3,4,5,6-Pentaphenyl-1-(1-methylquinolinium-3-yl)benzene methylsulfate (22b). According to Procedure 4, a solution of 
$0.100 \mathrm{~g}$ (0.171 mmol) of 2,3,4,5,6-pentaphenyl-1-(quinoline-3yl)benzene 10b, 1 drop of nitrobenzene and $0.06 \mathrm{~mL}$ $(0.63 \mathrm{mmol})$ of dimethyl sulfate in $5 \mathrm{~mL}$ of anhydrous toluene was heated for $2 \mathrm{~h}$ under reflux temperature to give 2,3,4,5,6pentaphenyl-1-(1-methylquinolinium-3-yl)benzene methylsulfate 22b. Yield 0.122 g, 99\%, a light green solid, m.p. $321{ }^{\circ} \mathrm{C}$ (decomp.). ${ }^{1} \mathrm{H}$ NMR (600 MHz, DMSO- $d_{6}$ ): $\delta=9.35$ (d, $J=1.4$ $\mathrm{Hz}, 1 \mathrm{H}, 2-\mathrm{H}), 8.84$ (s, 1H, 4-H), 8.24 (d, $J=8.1 \mathrm{~Hz}, 1 \mathrm{H}, 8-\mathrm{H})$, 8.14 (ddd, $J=1.5,7.1,8.8 \mathrm{~Hz}, 1 \mathrm{H}, 7-\mathrm{H}$ ), 8.06 (dd, $J=1.1,8.4$ $\mathrm{Hz}, 1 \mathrm{H}, 5-\mathrm{H}), 7.92-7.90(\mathrm{~m}, 1 \mathrm{H}, 6-\mathrm{H}), 7.05$ (d, $J=7.7 \mathrm{~Hz}, 2 \mathrm{H}$, $\mathrm{Ph}$ ), 7.02 (d, $J=7.7 \mathrm{~Hz}, 2 \mathrm{H}, \mathrm{Ph}), 6.96-6.82$ (m, 21H, Ph), 4.34 $\left(\mathrm{s}, 3 \mathrm{H}, \mathrm{NCH}_{3}\right), 3.37\left(\mathrm{~s}, 3 \mathrm{H}, \mathrm{CH}_{3} \mathrm{SO}_{4}\right) \mathrm{ppm} .{ }^{13} \mathrm{C} \mathrm{NMR}(150 \mathrm{MHz}$, DMSO- $\left.d_{6}\right): \delta=150.6(+, \mathrm{C} 2), 147.5(+, \mathrm{C} 4), 142.3$ (o), 140.7 (o), 140.5 (o), 139.3 (o), 139.0 (o), 138.4 (o), 135.53 (o, C8a), 135.48 (+, C7), 134.4 (o, C3), 132.8 (o, C1'), $131.0(+), 130.7(+), 130.64$ (+), $130.60(+), 130.54(+), 130.5$ (+, C6), 129.9 (+, C5), 127.7 (o, C4a), 127.4 (o), $126.89(+), 126.87(+), 126.80(+), 126.77(+)$, $126.4(+), 125.92(+), 125.85(+), 118.9(+, \mathrm{C} 8), 52.8\left(+, \mathrm{CH}_{3} \mathrm{SO}_{4}\right)$, $44.8\left(+, \mathrm{NCH}_{3}\right)$ ppm. IR (ATR): 3051, 3023, 2961, 1600, 1524, 1496, 1442, 1378, 1254, 1215, 1063, 1012, 760, 697, 618, 577, 558, $441 \mathrm{~cm}^{-1}$. HRMS (ESI): $\mathrm{m} / z$ calcd for $\mathrm{C}_{46} \mathrm{H}_{34} \mathrm{~N}[\mathrm{M}]^{+}$ 600.2686 , found 600.2687 .

2,3,4,5,6-Pentaphenyl-1-(1-methylquinolinium-4-yl)benzene methylsulfate (22c). According to Procedure 4, a solution of $0.154 \mathrm{~g}$ ( $0.263 \mathrm{mmol})$ of 2,3,4,5,6 pentaphenyl-1-(quinoline-4yl)benzene 10c, 1 drop of nitrobenzene and $0.06 \mathrm{~mL}$ $(0.63 \mathrm{mmol})$ of dimethyl sulfate in $5 \mathrm{~mL}$ of anhydrous toluene was heated for $2 \mathrm{~h}$ under reflux temperature to give $2,3,4,5,6$ pentaphenyl-1-(1-methylquinolinium-4-yl)benzene methylsulfate 22c. Yield $0.185 \mathrm{~g}, 99 \%$, a brownish solid, m.p. $355{ }^{\circ} \mathrm{C}$ (decomp.). ${ }^{1} \mathrm{H}$ NMR (600 MHz, DMSO- $\left.d_{6}\right): \delta=9.14(\mathrm{~d}, J=$ $6.0 \mathrm{~Hz}, 1 \mathrm{H}, 2-\mathrm{H}), 8.27$ (dd, $J=1.0,8.4 \mathrm{~Hz}, 1 \mathrm{H}, 5-\mathrm{H}), 8.20$ (d, $J=$ $8.9 \mathrm{~Hz}, 1 \mathrm{H}, 8-\mathrm{H}), 8.15$ (d, $J=6.0 \mathrm{~Hz}, 1 \mathrm{H}, 3-\mathrm{H}), 8.11$ (ddd, $J=$ 1.4, 7.1, 8.7 Hz, 1H, 7-H), 7.96-7.94 (m, 1H, 6-H), 7.06 (d, $J=$ $7.7 \mathrm{~Hz}, 2 \mathrm{H}, \mathrm{Ph}), 7.01$ (d, J = 7.7 Hz, 2H, Ph), 6.95-6.83 (m, 14H, $\mathrm{Ph})$, 6.74-6.71 (m, 4H, Ph), 6.60-6.57 (m, 2H, Ph), 4.42 (s, 3H, $\mathrm{NCH}_{3}$ ), 3.37 (s, 3H, $\mathrm{CH}_{3} \mathrm{SO}_{4}$ ) ppm. ${ }^{13} \mathrm{C}$ NMR (150 MHz, DMSO$\left.d_{6}\right): \delta=158.5$ (o, C4), 147.5 (+, C2), 142.3 (o), 140.6 (o), 139.36 (o), 139.34 (o), 138.9 (o), 138.3 (o), 137.1 (o, C8a), 135.2 (+, C7), 133.1 (o, C $\alpha), 131.0(+), 130.8(+), 130.52(+), 130.49(+), 129.83$ (+, C6), 128.80 (+), 129.3 (+, C5), 128.3 (o, C4a), 126.83 (+), $126.78(+), 126.74(+), 126.71(+), 126.67(+), 126.4(+)$, $125.81(+), 125.77(+), 125.3$ (+, C3), 118.8 (+, C8), 52.8 $\left(+, \mathrm{CH}_{3} \mathrm{SO}_{4}\right), 45.0\left(+, \mathrm{NCH}_{3}\right)$ ppm. IR (ATR): 3051, 3025, 1622, $1615,1599,1586,1577,1531,1495,1441,1401,1389,1375$, 1335, 1265, 1216, 1118, 1059, 1015, 869, 820, 766, 726, 695, $609,576,553,542,464,430 \mathrm{~cm}^{-1}$. HRMS (ESI): $\mathrm{m} / z$ calcd for $\mathrm{C}_{46} \mathrm{H}_{34} \mathrm{~N}[\mathrm{M}]^{+}$600.2686, found 600.2673.

1-(1-Methylquinolinium-3-yl)-2,3,4,5,-tetraphenyl-6-(4-(1,2,3,4tetraphenyl)-phenyl)benzene methylsulfate (23). According to Procedure 4, a solution of $0.481 \mathrm{~g}(0.5 \mathrm{mmol})$ of 1-(quinolin-3-yl)2,3,4,5,-tetraphenyl-6-(4-(1,2,3,4-tetraphenyl)phenyl)benzene $\mathbf{1 5}$, 1 drop of nitrobenzene and $0.06 \mathrm{~mL}(0.63 \mathrm{mmol})$ of dimethyl sulfate in $5 \mathrm{~mL}$ of anhydrous toluene was heated for $2 \mathrm{~h}$ under reflux temperature to give 1-(1-methylquinolinium-3-yl)2,3,4,5,-tetraphenyl-6-(4-(1,2,3,4-tetraphenyl)phenyl)benzene methylsulfate 23. Yield $0.521 \mathrm{~g}, 96 \%$, a khaki solid, m.p. $222{ }^{\circ} \mathrm{C}$ (decomp.). ${ }^{1} \mathrm{H}$ NMR (600 MHz, DMSO- $\left.d_{6}\right): \delta=9.28$ (d, $J=1.3 \mathrm{~Hz}, 1 \mathrm{H}, \mathrm{Q}), 8.73(\mathrm{~s}, 1 \mathrm{H}, \mathrm{Q}), 8.35$ (d, $J=6.7 \mathrm{~Hz}, 1 \mathrm{H}$, Q), 8.20 (ddd, $J=1.6,7.0,8.7 \mathrm{~Hz}, 1 \mathrm{H}, \mathrm{Q}), 8.05-8.04$ (m, 1H, Q), 7.98-7.95 (m, 1H, Q), 7.26-7.23 (m, 1H, Ph), 7.19-7.13 (m, 4H, $\mathrm{Ph}$ ), 7.02-6.99 (m, 3H, Ph), 6.95-6.74 (m, 28H, Ph), 6.71-6.64 (m, 5H, Ph), 6.61-6.53 (m, 3H, Ph), 6.49-6.47 (m, 1H, Ph), 4.33 $\left(\mathrm{s}, 3 \mathrm{H}, \mathrm{NCH}_{3}\right), 3.36\left(\mathrm{~s}, 3 \mathrm{H}, \mathrm{CH}_{3} \mathrm{SO}_{4}\right) \mathrm{ppm} .{ }^{13} \mathrm{C} \mathrm{NMR}(150 \mathrm{MHz}$, DMSO- $d_{6}$ ): $\delta=150.6(+), 147.5(+), 142.2$ (o), 141.5 (o), 140.9 (o), 140.7 (o), 140.6 (o), 140.4 (o), 140.3 (o), 140.0 (o), 139.6 (o), 139.3 (o), 139.01 (o), 139.00 (o), 138.96 (o), 138.8 (o), 138.6 (o), 138.4 (o), 137.3 (o), 136.5 (o), 135.6 (o), 135.5 (+), 134.4 (o), 132.8 (o), $131.0(+), 130.9(+), 130.8(+), 130.7(+), 130.64(+)$, $130.60(+), 130.55(+), 130.53(+), 130.44(+), 130.36(+)$, $130.28(+), 130.0(+), 129.4(+), 128.9(+), 128.6(+), 128.4(+)$, $128.2(+), 127.73(+), 127.66(+), 127.4(+), 126.85(+), 126.81(+)$, $126.51(+), 126.46(+), 126.4(+), 125.91(+), 125.85(+), 125.8(+)$, $125.44(+), 125.37(+), 125.31(+), 118.9(+), 52.7(+), 44.8(+)$ ppm. IR (ATR): 3055, 3023, 1600, 1524, 1442, 1378, 1249, 1222, 1178, 1157, 1138, 1058, 1008, 911, 852, 797, 766, 697, 565, 496, $432 \mathrm{~cm}^{-1}$. HRMS (ESI): $\mathrm{m} / z$ calcd for $\mathrm{C}_{76} \mathrm{H}_{54} \mathrm{~N}[\mathrm{M}]^{+}$980.4251, found 980.4257 .

1-(1-Methylquinolinium-3-yl)-2,3,4,5,-tetraphenyl-6-(4-(1,2,3,4tetraphenyl)phenyl)benzene hexafluorophosphate $\left(23 \mathrm{PF}_{6}\right)$. A suspension of $0.050 \mathrm{~g}(0.046 \mathrm{mmol})$ of 1-(1-methylquinolinium-3-yl)-2,3,4,5,-tetraphenyl-6-(4-(1,2,3,4-tetraphenyl) phenyl)benzene methylsulfate 23 and $0.008 \mathrm{~g}(0.049 \mathrm{mmol})$ of $\mathrm{NH}_{4} \mathrm{PF}_{6}$ in $4 \mathrm{~mL}$ of water was stirred for 1 day at rt to give 1-(1methylquinolinium-3-yl)-2,3,4,5,-tetraphenyl-6-(4-(1,2,3,4-tetraphenyl)phenyl)benzene hexafluorophosphate $\mathbf{2 3 P F}_{\mathbf{6}}$. Yield $0.049 \mathrm{~g}, 95 \%$, a yellow solid, m.p. $220{ }^{\circ} \mathrm{C}$ (decomp.). ${ }^{1} \mathrm{H}$ NMR $\left(600 \mathrm{MHz}, \mathrm{DMSO}-d_{6}\right): \delta=9.28(\mathrm{~s}, 1 \mathrm{H}, \mathrm{Q}), 8.73$ (s, $\left.1 \mathrm{H}, \mathrm{Q}\right), 8.35$ (d, $J=8.9 \mathrm{~Hz}, 1 \mathrm{H}, \mathrm{Q}), 8.20$ (t, $J=7.7 \mathrm{~Hz}, 1 \mathrm{H}, \mathrm{Q}), 8.05$ (d, $J=7.9$ $\mathrm{Hz}, 1 \mathrm{H}, \mathrm{Q}), 7.96$ (t, $J=7.5 \mathrm{~Hz}, 1 \mathrm{H}, \mathrm{Q}), 7.26(\mathrm{~m}, 1 \mathrm{H}, \mathrm{Ph})$, 7.17-7.14 (m, 4H, Ph), 7.02-6.47 (m, 40H, Ph), $4.33(\mathrm{~s}, 3 \mathrm{H}$, $\mathrm{NCH}_{3}$ ) ppm. ${ }^{13} \mathrm{C}$ NMR (150 MHz, DMSO- $\left.d_{6}\right): \delta=150.6(+)$, 147.5 (+), 142.2 (o), 141.5 (o), 140.9 (o), 140.7 (o), 140.6 (o), 140.4 (o), 140.3 (o), 140.0 (o), 139.6 (o), 139.3 (o), 139.01 (o), 139.00 (o), 138.96 (o), 138.8 (o), 138.6 (o), 138.4 (o), 137.3 (o), 136.5 (o), 135.6 (o), 135.5 (+), 134.4 (o), 132.8 (o), 131.0 (+), $130.9(+), 130.8(+), 130.7(+), 130.64(+), 130.60(+), 130.55(+)$, $130.53(+), 130.44(+), 130.36(+), 130.28(+), 130.0(+), 129.4(+)$, $128.9(+), 128.6(+), 128.4(+), 128.2(+), 127.73(+), 127.66(+)$, $127.4(+), 126.85(+), 126.81(+), 126.51(+), 126.46(+), 126.4(+)$, $125.91(+), 125.85(+), 125.8(+), 125.44(+), 125.37(+), 125.31$ $(+), 118.9(+), 44.8(+)$ ppm. IR (ATR): 3055, 3027, 1600, 1521, 1495, 1442, 1379, 1072, 1028, 835, 766, 697, 557, $427 \mathrm{~cm}^{-1}$. HRMS (ESI): $m / z$ calcd for $\mathrm{C}_{76} \mathrm{H}_{54} \mathrm{~N}[\mathrm{M}]^{+}$980.4251, found 980.4238 .

1,2-Di(1-methylquinolinium-3-yl)-3,4,5,6-tetraphenylbenzene dihexafluorophosphate (24a). A solution of $0.050 \mathrm{~g}$ (0.079 mmol) of 1,2-di(quinoline-3-yl)-3,4,5,6-tetraphenylbenzene 19a, 1 drop of nitrobenzene and $0.02 \mathrm{~mL}(0.21 \mathrm{mmol})$ of dimethyl sulfate in $5 \mathrm{~mL}$ of anhydrous toluene was heated for $3 \mathrm{~h}$ under reflux temperature, cooled to rt and extracted with water $(3 \times 5 \mathrm{~mL})$ and precipitated with excess of $\mathrm{NH}_{4} \mathrm{PF}_{6}$ 
(1.3 equiv.) to give 1,2-di(1-methylquinolinium-3-yl)-3,4,5,6-tetraphenylbenzene dihexafluorophosphate 24a. Yield $0.068 \mathrm{~g}$, $90 \%$, a white solid, m.p. $240{ }^{\circ} \mathrm{C} .{ }^{1} \mathrm{H}$ NMR $(600 \mathrm{MHz}$, DMSO- $\left.d_{6}\right)$, two sets of isochronous rotameric forms $(0.9: 1)$.: $\delta=9.43(\mathrm{~d}, J=1.3 \mathrm{~Hz}, 0.9 \mathrm{H}, 2-\mathrm{H}), 9.40\left(\mathrm{~d}, J=1.3 \mathrm{~Hz}, 1 \mathrm{H}, 2^{\prime}-\mathrm{H}\right)$, $9.05\left(\mathrm{~s}, 1 \mathrm{H}, 4^{\prime}-\mathrm{H}\right), 8.88(\mathrm{~s}, 0.9 \mathrm{H}, 4-\mathrm{H}), 8.30(\mathrm{~d}, J=8.8 \mathrm{~Hz}, 1 \mathrm{H}$, $\left.8^{\prime}-\mathrm{H}\right), 8.27\left(\mathrm{~d}, J=8.9 \mathrm{~Hz}, 0.9 \mathrm{H}, 8^{\prime}-\mathrm{H}\right), 8.16-8.09(\mathrm{~m}, 3.8 \mathrm{H}, 5-\mathrm{H}$, $\left.5^{\prime}-\mathrm{H}, 7-\mathrm{H}, 7^{\prime}-\mathrm{H}\right), 7.93-7.90\left(\mathrm{~m}, 1.9 \mathrm{H}, 6-\mathrm{H}, 66^{\prime}-\mathrm{H}\right), 7.05-6.84$ (m, $48 \mathrm{H}, \mathrm{Ph}), 4.38\left(\mathrm{~s}, 6 \mathrm{H}, \mathrm{N}^{\prime} \mathrm{CH}_{3}\right), 4.32\left(\mathrm{~s}, 5.4 \mathrm{H}, \mathrm{NCH}_{3}\right) \mathrm{ppm} .{ }^{13} \mathrm{C}$ NMR (150 MHz, DMSO- $\left.d_{6}\right): \delta=150.6(+, \mathrm{C} 2), 149.8\left(+, \mathrm{C} 2{ }^{\prime}\right)$, 147.9 (+, C4'), 147.6 (+, C4), 142.8 (o), 142.7 (o), 141.9 (o), 141.8 (o), 138.5 (o), 137.6 (o), 136.5 (o, C8a'), 136.4 (o, C8a), 135.94 (+, C7 or $\mathrm{C7}^{\prime}$ ), 135.85 (+, C7 or $\mathrm{C7}^{\prime}$ ), 133.10 (o, C $\left.\beta\right), 132.99$ (o, C $\alpha), 132.4$ (o), 132.2 (o), 130.89 (+), $130.75(+), 130.69(+)$, $130.60(+), 130.48(+), 130.44(+), 130.42(+), 130.38(+), 130.33$ (+), $130.31(+), 130.22$ (+, C5), 130.07 (+, C5'), 127.92 (o, C4a'), 127.89 (o, C4a), $127.6(+), 127.0(+), 126.7(+), 126.3(+), 119.00$ $\left(+, \mathrm{C}^{\prime}\right), 118.90(+, \mathrm{C} 8), 45.4\left(+, \mathrm{N}^{\prime} \mathrm{CH}_{3}\right), 45.2\left(+, \mathrm{NCH}_{3}\right) \mathrm{ppm} . \mathrm{IR}$ (ATR): 3057, 3027, 1631, 1602, 1583, 1523, 1497, 1443, 1380, 1356, 1334, 1230, 1174, 1141, 1115, 1073, 1024, 936, 830, 769, 744, 702, 619, 556, 524, 504, $419 \mathrm{~cm}^{-1}$. HRMS (ESI): $\mathrm{m} / z$ calcd for $\mathrm{C}_{50} \mathrm{H}_{38} \mathrm{~N}_{2}[\mathrm{M}]^{2+}$ 333.1512, found 333.1518.

1-(1-Methylquinolinium-3-yl)-2-(1-methylquinolinium -4-yl)3,4,5,6-tetraphenylbenzene dihexafluorophosphate (24b). A solution of $0.050 \mathrm{~g}(0.079 \mathrm{mmol})$ of 1-(quinoline-3-yl)-2-(quinoline-4-yl)-3,4,5,6-tetraphenylbenzene 19b, 1 drop of nitrobenzene and $0.02 \mathrm{~mL}(0.21 \mathrm{mmol})$ of dimethyl sulfate in $5 \mathrm{~mL}$ of anhydrous toluene was heated for $3 \mathrm{~h}$ under reflux temperature, cooled to rt and extracted with water $(3 \times 5 \mathrm{~mL})$ and then precipitated with excess of $\mathrm{NH}_{4} \mathrm{PF}_{6}$ (1.3 equiv.) to give 1-(1methylquinolinium-3-yl)-2-(1-methylquinolinium-4-yl)-3,4,5,6tetraphenylbenzene dihexafluorophosphate 24b. Yield $0.067 \mathrm{~g}$, $89 \%$, a yellow solid, m.p. $245{ }^{\circ} \mathrm{C}$ (decomp.). ${ }^{1} \mathrm{H}$ NMR $\left(600 \mathrm{MHz}\right.$, DMSO- $\left.d_{6}\right)$, two sets of isochronous rotameric forms $\left(\mathbf{2 4} \mathbf{b}^{*} / \mathbf{2 4} \mathbf{b}^{\#}=1: 1.27\right)$ with close chemical shifts, exact description of the coupling constants is not possible: $\delta=9.54^{\#}(\mathrm{~d}, J=$ $1.3 \mathrm{~Hz}, 1 \mathrm{H}, 2-\mathrm{H}), 9.23^{*}(\mathrm{~d}, J=1.5 \mathrm{~Hz}, 1 \mathrm{H}, 2-\mathrm{H}), 9.20^{\#}(\mathrm{~d}, J=$ $\left.6.3 \mathrm{~Hz}, 1 \mathrm{H}, 2^{\prime}-\mathrm{H}\right), 9.18^{*}\left(\mathrm{~d}, J=6.4 \mathrm{~Hz}, 1 \mathrm{H}, 2^{\prime}-\mathrm{H}\right), 9.02^{*}(\mathrm{~d}, J=$ $1.5 \mathrm{~Hz}, 1 \mathrm{H}, 4-\mathrm{H}), 8.81^{\#}(\mathrm{~s}, 1 \mathrm{H}, 4-\mathrm{H}), 8.34^{\#}(\mathrm{dd}, J=1.1,8.6 \mathrm{~Hz}$, $1 \mathrm{H}), 8.26-8.20(\mathrm{~m}, 6 \mathrm{H}), 8.16^{\#}\left(\mathrm{~d}, J=6.3 \mathrm{~Hz}, 1 \mathrm{H}, 3^{\prime}-\mathrm{H}\right)$, 8.15-8.07 (m, 7H), 8.04-8.01 $(\mathrm{m}, 2 \mathrm{H}), 7.92^{*}(\mathrm{ddd}, J=1.2,6.8$, $8.1 \mathrm{~Hz}, 1 \mathrm{H}), 7.85-7.79^{\#}(\mathrm{~m}, 2 \mathrm{H}), 7.21-6.62^{* \#}(\mathrm{~m}, 40 \mathrm{H}, \mathrm{Ph})$, $4.37^{\#}\left(\mathrm{~s}, 3 \mathrm{H}, \mathrm{N}^{\prime} \mathrm{CH}_{3}\right), 4.346^{\#}\left(\mathrm{~s}, 3 \mathrm{H}, \mathrm{NCH}_{3}\right), 4.343^{*}\left(\mathrm{~s}, 3 \mathrm{H}, \mathrm{N}^{\prime}\right.$ $\left.\mathrm{CH}_{3}\right), 4.10^{*}\left(\mathrm{~s}, 3 \mathrm{H}, \mathrm{NCH}_{3}\right) \mathrm{ppm} .{ }^{13} \mathrm{C}$ NMR (150 MHz, DMSO$\left.d_{6}\right): \delta=155.64^{* / \#}\left(\mathrm{o}, \mathrm{C} 4^{\prime}\right), 155.59^{* / \#}\left(\mathrm{o}, \mathrm{C} 4^{\prime}\right), 149.53^{\#}(+, \mathrm{C} 2)$, 149.31* $(+, \mathrm{C} 2), 148.47^{\#}\left(+, \mathrm{C} 2{ }^{\prime}\right), 148.42^{*}\left(+, \mathrm{C} 2^{\prime}\right), 147.14^{*}(+, \mathrm{C} 4)$, 146.81 ${ }^{\#}$ (+, C4), 142.92 (o), 142.90 (o), 142.89 (o), 142.84 (o), 141.63 (o), 141.49 (o), 140.37 (o), 140.21 (o), 138.56 (o), 138.48 (o), 138.35 (o), 138.31 (o), 137.54 (o), 137.52 (o), 137.48 (o), 137.43 (o), $136.38^{\#}$ (o, C8a), 136.10* (o, C8a), $135.96(+), 135.88(+)$, $135.46(+), 135.38(+), 133.36^{*}(\mathrm{o}, \mathrm{C} \beta), 133.23^{\#}(\mathrm{o}, \mathrm{C} \beta), 132.26(\mathrm{o})$, 132.21 (o), 132.05 (o), 131.97 (o), $131.16(+), 130.88(+), 130.73(+)$, $130.63(+), 130.61(+), 130.58(+), 130.55(+), 130.51(+), 130.42(+)$, $130.30(+), 130.26(+), 130.23(+), 130.22(+), 129.71(+), 129.63(+)$, $129.50(+), 128.60(+), 128.32(+), 127.88(0), 127.71(0), 127.61(+)$, $127.59(+), 127.44(+), 127.37(+), 127.15(+), 127.07(+), 127.03(+)$,
$126.98(+), 126.93(+), 126.85(+), 126.79(+), 126.64(+), 126.30(+)$, $126.26(+), 126.24(+), 125.24(+), 124.78(+), 119.34(+), 119.02(+)$, $118.86(+), 45.37(+), 45.33(+), 45.30(+), 44.67^{*}\left(+, \mathrm{NCH}_{3}\right) \mathrm{ppm}$. IR (ATR): 3057, 1619, 1605, 1588, 1529, 1498, 1443, 1234, 1175, 1115, 1073, 1025, 1001, 918, 827, 765, 702, 619, 556, 492, $434 \mathrm{~cm}^{-1}$. HRMS (ESI): $\mathrm{m} / z$ calcd for $\mathrm{C}_{50} \mathrm{H}_{38} \mathrm{~N}_{2}[\mathrm{M}]^{2+} 333.1512$, found 333.1512.

Hexakis(1-methylquinolinium-3-yl)benzene hexakis hexafluorophosphate (25). According to Procedure 4, a solution of $0.084 \mathrm{~g}$ (0.10 mmol) of hexakis(quinoline-3-yl)benzene 21, 1 drop of nitrobenzene and $0.10 \mathrm{~mL}(1.00 \mathrm{mmol})$ of dimethyl sulfate in $5 \mathrm{~mL}$ of anhydrous toluene was heated for $3 \mathrm{~h}$ under reflux temperature. Then the obtained salt was dissolved in water and precipitated with 1.05 equiv. of $\mathrm{NH}_{4} \mathrm{PF}_{6}$ to give hexakis(1-methylquinolinium-3-yl)benzene hexakis-hexafluorophosphate 25. Yield $0.171 \mathrm{~g}, 95 \%$, a white solid, m.p. $195{ }^{\circ} \mathrm{C}$ (decomp.). ${ }^{1} \mathrm{H}$ NMR (600 MHz, DMSO- $\left.d_{6}\right): \delta=9.41-8.89(\mathrm{~m}$, $12 \mathrm{H}), 8.34-8.16(\mathrm{~m}, 18 \mathrm{H}), 8.00-7.92(\mathrm{~m}, 6 \mathrm{H}), 4.44-4.28(\mathrm{~m}$, $18 \mathrm{H})$ ppm. ${ }^{13} \mathrm{C}$ NMR (150 MHz, DMSO- $\left.d_{6}\right): \delta=148.7,148.2$, $137.9,137.8,137.7,137.5,137.0,131.0,130.4,128.6,128.0$, 119.4, 45.97, 45.89, 45.85, 45.79 ppm. IR (ATR): 3084, 1631, 1582, 1524, 1451, 1382, 1228, 1173, 1040, 952, 920, 827, 771, $753,740,614,556,494,440,413 \mathrm{~cm}^{-1}$.

\section{Conflicts of interest}

There are no conflicts to declare.

\section{References}

1 F. J. Schmitz, K. H. Hollenbeak and D. C. Campbell, J. Org. Chem., 1978, 43, 3916.

2 (a) N. Fusetani, N. Asai and S. Matsunaga, Tetrahedron Lett., 1994, 23, 3967; (b) H. Anan, N. Seki, O. Noshiro, K. Hona, K. Yasumuro, T. Ozasa and N. Fusetani, Tetrahedron, 1996, 52, 10849.

3 S. Albrizio, P. Ciminiello, E. Fattorusso, S. Magno and J. R. Pawlik, J. Nat. Prod., 1995, 58, 647.

4 C. A. Volk and M. Köck, Org. Lett., 2003, 5, 3567.

5 C. A. Volk and M. Köck, Org. Biomol. Chem., 2004, 2, 1827.

6 R. Laville, O. P. Thomas, F. Berrue, F. Reyes and P. Amade, Eur. J. Org. Chem., 2008, 121.

7 (a) K. A. Smith and A. Streitwieser Jr., J. Org. Chem., 1983, 48, 2629; (b) K. C. Waterman and A. Streitwieser Jr., J. Am. Chem. Soc., 1984, 106, 3874; (c) K. A. Smith, K. C. Waterman and A. Streitwieser Jr., J. Org. Chem., 1985, 50, 3360; (d) K. C. Waterman, D. V. Speer, A. Streitwieser Jr., G. C. Look, K. O. Nguyen and J. G. Stack, J. Org. Chem., 1988, 53, 583; (e) A. S. Koch, K. C. Waterman, K. Banks and A. Streitwieser, J. Org. Chem., 1990, 55, 6166; $(f)$ A. S. Feng, D. V. Speer, S. G. DiMagno, M. S. Konings and A. Streitwieser, J. Org. Chem., 1992, 57, 2902.

8 A. S. Koch, A. S. Feng, T. A. Hopkins and A. Streitwieser, J. Org. Chem., 1993, 58, 1409. 
9 F. G. Pühlhofer and R. Weiss, Eur. J. Org. Chem., 2004, 1002.

10 (a) R. Weiss, R. Roth, R. H. Lowack and H. Bremer, Angew. Chem., 1990, 102, 1164, (Angew. Chem., Int. Ed. Engl., 1990, 29, 1132); (b) R. Weiss, B. Pomrehn, F. Hampel and W. Bauer, Angew. Chem., 1995, 107, 1446, (Angew. Chem., Int. Ed. Engl., 1995, 34, 1319); (c) R. Weiss, R. May and B. Pomrehn, Angew. Chem., 1996, 108, 1319, (Angew. Chem., Int. Ed. Engl., 1996, 35, 1232); (d) R. Weiss, S. M. Huber and F. G. Pühlhofer, Eur. J. Org. Chem., 2005, 3530; (e) R. Weiss, S. M. Huber, F. W. Heinemann, P. Audebert and F. G. Pühlhofer, Angew. Chem., 2006, 118, 8228, (Angew. Chem. Int. Ed., 2006, 45, 8059); (f) R. Weiss and F. G. Pühlhofer, J. Am. Chem. Soc., 2007, 129, 547.

11 (a) A. Schmidt, T. Mordhorst and M. Nieger, Synthesis, 2006, 3987; (b) A. Schmidt, J. C. Namyslo and T. Mordhorst, Tetrahedron, 2006, 62, 6893; (c) A. Schmidt, T. Mordhorst and M. Nieger, Tetrahedron, 2006, 62, 1667; (d) A. Schmidt and T. Mordhorst, Synthesis, 2005, 781; (e) A. Schmidt, J. Heterocycl. Chem, 2002, 39, 949.

12 (a) A. Schmidt and A. Hetzheim, Tetrahedron, 1997, 53, 1295; (b) A. Schmidt and M. K. Kindermann, J. Org. Chem., 1998, 63, 4636; (c) A. Schmidt and M. Nieger, J. Chem. Soc., Perkin Trans. 1, 1999, 1325.

13 A. Schmidt and T. Mordhorst, Heterocycles, 2006, 68, 1393.

14 (a) A. Schmidt, T. Mordhorst and M. Nieger, Org. Biomol. Chem., 2005, 3, 3788; (b) A. Schmidt, T. Mordhorst and T. Habeck, Org. Lett., 2002, 4, 1375.

15 (a) S. G. DiMagno, K. C. Waterman, D. V. Speer and A. Streitwieser, J. Am. Chem. Soc., 1991, 113, 4679; (b) A. S. Koch, A. S. Feng, T. A. Hopkins and A. Streitwieser, J. Org. Chem., 1993, 58, 1409.

16 (a) K. Akiyama, S. Tero-Kubota and Y. Ikegami, J. Am. Chem. Soc., 1983, 105, 3601; (b) Y. Ikegami, T. Muramatsu and K. Hanaya, J. Am. Chem. Soc., 1989, 111, 5782; (c) K. Okada, K. Matsumoto, M. Oda, H. Murai, K. Akiyama and Y. Ikegami, Tetrahedron Lett., 1995, 36, 6689.

17 R. Weiss, R. May and B. Pohmrehn, Angew. Chem., 1996, 108, 1319, (Angew. Chem., Int. Ed. Engl., 1996, 35, 1232).

18 (a) W. R. Boon, Chem. Ind., 1965, 782; (b) A. L. Black and L. A. Summers, J. Chem. Soc. C, 1969, 610; (c) E. C. Campbell, E. E. Glover and G. Trenholm, J. Chem. Soc., 1969, 1987; (d) A. D. Dodge, Endeavour, 1970, 111, 130.

19 (a) C. N. Corder and J. L. Way, J. Med. Chem., 1966, 9, 638; (b) Y. Ashani and S. Cohen, J. Med. Chem., 1971, 14, 621; (c) C. F. Barfknecht, F. W. Benz and J. P. Long, J. Med. Chem., 1971, 14, 1003.

20 M. Komloova, K. Musilek, A. Horova, O. Holas, V. Dohnal, F. Gunn-Moore and K. Kuca, Bioorg. Med. Chem. Lett., 2011, 21, 2505.

21 J. Hiller, M. Liu and A. Schmidt, Heterocycles, 2017, 94, 821.

22 M. Drev, U. Grošelj, B. Ledinek, F. Perdih, J. Svete, B. Štefane and F. Požgan, Org. Lett., 2018, 20, 5268.

23 L. E. Harrington, J. F. Britten, K. Nikitin and M. J. McGlinchey, ChemPlusChem, 2017, 82, 433.
24 Y. Geng, A. Fechtenkötter and K. Müllen, J. Mater. Chem., 2001, 11, 1634.

25 K. Kobayashi, A. Sato, S. Sakamoto and K. Yamaguchi, J. Am. Chem. Soc., 2003, 125, 3035.

26 S. Hiraoka, T. Nakamura, M. Shiro and M. Shionoya, J. Am. Chem. Soc., 2010, 132, 13223.

27 Z. Tomović, J. van Dongen, S. J. George, H. Xu, W. Pisula, P. Leclère, M. M. J. Smulders, S. De Feyter, E. W. Meijer and A. P. H. J. Schenning, J. Am. Chem. Soc., 2007, 129, 16190.

28 S. Hiraoka, Y. Hisanaga, M. Shiro and M. Shionoya, Angew. Chem., 2010, 122, 1713, (Angew. Chem., Int. Ed., 2010, 49, 1669).

29 B. Traber, J. J. Wolff, F. Rominger, T. Oeser, R. Gleiter, M. Goebel and R. Wortmann, Chem. - Eur. J., 2004, 10, 1227.

30 R. Shukla, S. V. Lindeman and R. Rathore, J. Am. Chem. Soc., 2006, 128, 5328.

31 M. Steeger and C. Lambert, Chem. - Eur. J., 2012, 18, 11937.

32 Y. Tanaka, T. Koike and M. Akita, Chem. Commun., 2010, 46, 4529.

33 S. H. Shin and N. Y. Sim, Korean Kongkae Taeho Kongbo, KR2016072868A20160624, 2016.

34 (a) D. Gust, J. Am. Chem. Soc., 1977, 99, 6980; (b) J. C. J. Bart, Acta Crystallogr., Sect. B: Struct. Sci., 1968, 24, 1277; (c) A. Almenningen, O. Bastiansen and P. N. Skancke, Acta Chem. Scand., 1958, 12, 1215.

35 G. Hilt, T. Vogler, W. Hess and F. Galbiati, Chem. Commun., 2005, 11, 1474.

36 (a) M. Mazik, D. Bläser and R. Boese, Tetrahedron Lett., 2000, 41, 5827; (b) R. Taylor and O. Kennard, J. Am. Chem. Soc., 1982, 104, 5063; (c) F. A. Cotton, L. M. Daniels, G. T. Jordan IV and C. A. Murillo, Chem. Commun., 1997, 1673; (d) G. R. Desiraju, Crystal Engineering. The Design of Organic Solids, Elsevier, Amsterdam, 1989; p. 166.

37 (a) K. Ghosh and A. J. Bhattacharya, Indian J. Chem., Sect. B: Org. Chem. Incl. Med. Chem., 1977, 15, 678; (b) P. K. Banerjee, S. R. Samanta and A. J. Bhattacharya, Curr. Sci., 1976, 45, 254.

38 E. Gagnon, T. Maris, P.-M. Arseneault, K. E. Maly and J. D. Wuest, Cryst. Growth Des., 2010, 10, 648.

39 E. Gagnon, S. D. Halperin, V. Métivaud, K. E. Maly and J. D. Wuest, J. Org. Chem., 2010, 75, 399.

40 D. Gust and A. Patton, J. Am. Chem. Soc., 1978, 100, 8175.

41 (a) M. A. Ogliaruso, M. G. Romanelli and E. I. Becker, Chem. Rev., 1965, 65, 261; (b) S. Saito and Y. Yamamoto, Chem. Rev., 2000, 10, 2901.

42 (a) J. H. Magill and A. R. Ubbelohde, Trans. Faraday Soc., 1958, 54, 1811; (b) D. J. Plazek and J. H. Magill, J. Chem. Phys., 1966, 45, 3038.

43 D. Wasserfallen, G. Mattersteig, V. Enkelmann and K. Müllen, Tetrahedron, 2006, 62, 5417.

44 H. D. Katz, J. Org. Chem., 1987, 52, 3932.

45 (a) A. Schmidt, S. Batsyts, A. Smeyanov, T. Freese, E. G. Hübner and M. Nieger, J. Org. Chem., 2016, 81, 4202; (b) S. Batsyts, F. J. Ramírez, J. Casado, J. C. Namyslo and 
A. Schmidt, Z. Naturforsch., 2018, 73, 481; (c) A. Dreger, R. Cisneros Camuña, N. Münster, T. A. Rokob, I. Pápai and A. Schmidt, Eur. J. Org. Chem., 2010, 4296; (d) M. Liu, M. Nieger, E. Hübner and A. Schmidt, Chem. - Eur. J., 2016, 22, 5416 .

46 S. Batsyts, R. Vedmid, J. C. Namyslo, M. Nieger and A. Schmidt, Eur. J. Org. Chem., 2019, 1301.

47 M. S. El-Shall and K. P. C. Vollhardt, J. Mol. Struct.: THEOCHEM, 1989, 183, 175.

48 A. A. Granovsky, Firefly version 8, http://www.classic.chem. msu.su/gran/firefly/index.html.
49 M. W. Schmidt, K. K. Baldridge, J. A. Boatz, S. T. Elbert, M. S. Gordon, J. H. Jensen, S. Koseki, N. Matsunaga, K. A. Nguyen, S. Su, T. L. Windus, M. Dupuis and J. A. Montgomery, J. Comput. Chem., 1993, 14, 1347.

50 E. D. Glendening, J. K. Badenhoop, A. E. Reed, J. E. Carpenter, J. A. Bohmann, C. M. Morales and F. Weinhold, NBO 5.9, Theoretical Chemistry Institute, University of Wisconsin, Madison, WI, 2012.

51 M.-H. Son, J. Y. Kim, E. J. Lim, D.-J. Beak, K. Choi, J. K. Lee, A. N. Pee, S.-J. Min and Y. S. Cho, Bioorg. Med. Chem. Lett., 2013, 23, 1472. 